\title{
Combinatorial representation of tetrahedral chains
}

\author{
Hassan Babiker and StanisŁaw Janeczko
}

\begin{abstract}
Tetrahedral chains consisting of face-sharing regular tetrahedra in Euclidean three space are investigated. A parametrization of the chains by sequences of ordered reflections in barycentric coordinates is constructed and periodicity in their combinatorial structure is found. Periodicity along a chain is based on the structure of sequences of admissible triplets of integers and their cycling properties. The corresponding numerical invariants and an indexing role of a binary tetrahedral group are established.
\end{abstract}

\section{Introduction}

The simplest naturally ordered tetrahedral packing consists of an ordered sequence of regular tetrahedra glued together face to face as in the linear packing of a tetrahedral helix (Figure 1) introduced by H.S.M. Coxeter in [1. Such tetrahedral structures, have been studied already by several authors [8]10, 12], are called tetrahedral chains.

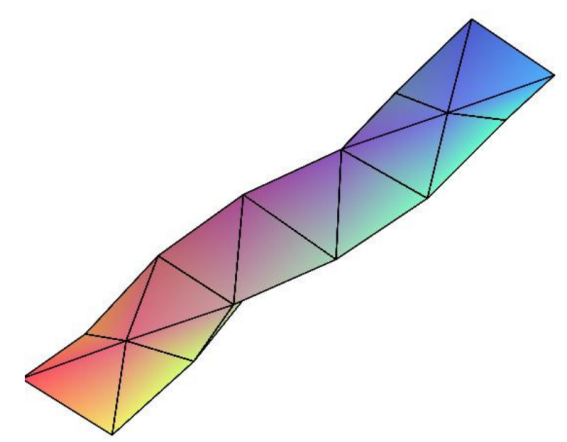

Figure 1: Tetrahelix.

Partially supported by NCN grant no. DEC-2013/11/B/ST1/03080. 
Any tetrahedral chain consists of three types of simplest configurations of four consecutive tetrahedra called tetrahedral units. Two of these types are left and right tetrahedral short spirals, $U, D$, and the third type, $F$, is a flat configuration of four tetrahedra (Figure 2). The structure of a tetrahedral chain in $D, F, U$ elementary units is written as a word like $U U D F U D \cdots$.

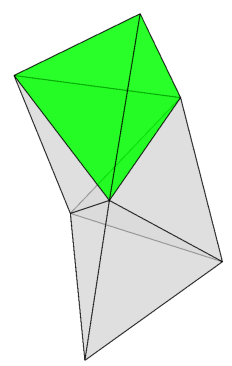

D

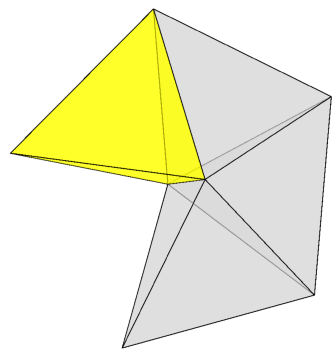

F

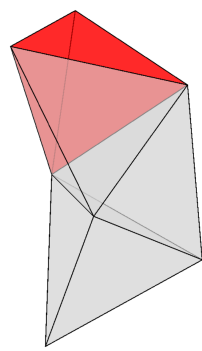

$\mathrm{U}$

Figure 2: Tetrahedral units.

The three strands of the left or right oriented tetrahedral helix form a spiral with irrational slope. This is the reason for the effective density of tetrahedral chains (as proved by J.H.Mason [9]) and nonexistence of closed tetrahedral chains in Euclidean space (Figure 3 ).

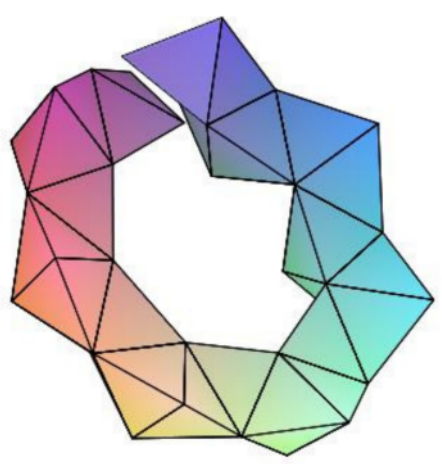

Figure 3: Semi-ring structure.

Let us assume that the gluing process of tetrahedra is ordered along a chain and each step of this process is realized by reflection in a particular 
face of adjacent tetrahedron. To each tetrahedron we assign four reflections $R_{i}, i=1, \ldots, 4$, in the configurational three dimensional space $V$. Reflections $R_{i}$ in $V$ are represented by four corresponding reflect-morphisms $\bar{R}_{i}, i=$ $1, \ldots, 4$, acting in the space of regular tetrahedra $\mathcal{T}$ through a reflectional transformation of their vertices. Any tetrahedral chain of length $n+1$ is uniquely represented by an initial tetrahedron $T$ and an ordered sequence of $n$ reflect-morphisms

$$
\bar{R}_{i_{1}}, \ldots, \bar{R}_{i_{n}}, \quad i_{k} \neq i_{k+1}, \quad k=1, \ldots, n-1
$$

The fact that a tetrahedral chain is so rigid in 3-space and regular tetrahedra can not tile the space gives rise to several questions. The main question which we consider in this paper is the recognition of combinatorial and algebraic structures of tetrahedral chains. We want to investigate their geometric properties and determine the kind of shape information which is contained in the chain invariants of orthogonal transformations and re-numberings (cf. [8, 13]).

In Section 2 we introduce regular tetrahedra and affine reflections in their faces. Regular tetrahedra defined by four vertices are identified with elements of the tensor product of configurational three space and the four dimensional space of barycentric coordinates. Replacing all affine reflections of tetrahedra in faces by four linear reflections - reflect-morphisms, in barycentric four dimensional space, we uniquely encode all $D, F, U$ elements of a chain by triplets of consecutive reflect-morphisms. Fixing enumeration of an initial tetrahedron (its vertices) we find that a reflect-morphism which reconstructs the adjacent tetrahedron by reflection along a chain, is denoted by the corresponding number of the reflected vertex. This way in Section 3 we parameterize a tetrahedral chain by the sequence of admissible triplets of integers $(k, j, i), k \neq j \neq i, 1 \leq k, j, i \leq 4$ and translate its geometric structure to purely combinatorial invariants of the re-numbering of the initial data. Thus the geometrical shape information of the chain is encoded by the sequence of admissible oriented triplets of integers displayed in Table 1. In Section 4 we investigate combinatorial properties of sequences of admissible triplets of integers and discuss notions of equivalence of representations. In particular we show that a complete invariant of chain equivalence is the sequence of signs $\{-1,0,+1\}$ to which we reduce an admissible chain. In Section 5all proper chains, i.e. chains which do not self-intersect, until their length equals 13 are listed and all proper chains centered around one common vertex with various branching orders are classified. There were found six proper chains centered around one vertex - icosi-clusters of maximal 
length $n=20$ and branching order $b=1$. The periodic chains are investigated in Section 6 where it is proved that any periodic tetrahedral chain can be reconstructed by the composition of reflect-morphisms $M$ and their powers $M^{m}$. The tetrahedral chains as sequences of $D, F, U$ are replaced by compositions of the three corresponding permutations $\mathcal{L}_{u}, \mathcal{L}_{d}, \mathcal{L}_{f}$ of 12 elements set $\mathbb{I}=\left\{(\alpha, \beta) \in \Delta_{4} \times \Delta_{4}: \alpha \neq \beta\right\}, \Delta_{4}=\{1,2,3,4\}$. It is shown, that the permutations $\mathcal{L}_{u}, \mathcal{L}_{d}, \mathcal{L}_{f}$ of $\mathbb{I}$ generate a tetrahedral group representation. And the non-periodic tetrahedral chains are indexed by elements of the tetrahedral group.

\section{Tetrahedra in barycentric coordinates}

A regular tetrahedron $T$ in Euclidean 3-space is identified with the set of its four vertices $\left\{p_{1}, p_{2}, p_{3}, p_{4}\right\}, p_{i} \in \mathbb{R}^{3}$. We decompose $T$ into four faces; $T=S_{1} \cup \cdots \cup S_{4}$, where we enumerate faces in such a way that the vertex $p_{i}$ lies outside the plane defined by the face $S_{i}$. By $\left(S_{i}, p_{i}\right)$ we denote an elementary pair of $T$. We also write $T=\left\{\left(S_{1}, p_{1}\right), \ldots,\left(S_{4}, p_{4}\right)\right\}$. We denote by $\mathcal{T}$ the space of regular tetrahedra in $\mathbb{R}^{3}$, and in what follows we only consider regular tetrahedra. Let $\left(p_{1}, p_{2}, p_{3}, p_{4}\right)$ be vertices of the tetrahedron $T$. We identify the space $\mathcal{T}$ of regular tetrahedra with a subspace of the tensor product $\mathcal{T} \subset V \otimes U^{*}, U \equiv \mathbb{R}^{4}$, where $V$ is a configurational vector space, $\operatorname{dim} V=3$, and $U$ is a space of barycentric coordinates $\left(\alpha_{1}, \ldots, \alpha_{4}\right) \in$ $\mathbb{R}^{4}$ contained in the canonical affine hyperplane $H=\left\{\sum_{i=1}^{4} \alpha_{i}=1\right\}$. Each tetrahedron $T \in \mathcal{T}$ defines the barycentric tetrahedral coordinates on $V$. For a regular tetrahedron $T \in \mathcal{T}$ we write $T=\sum_{i=1}^{4} p_{i} \otimes e_{i}^{*}$, where $\left\{p_{i}\right\}$ satisfies the regularity equations, $\left\|p_{i}-p_{j}\right\|=\left\|p_{k}-p_{l}\right\|$ for $i \neq j, k \neq l$ and $e_{i}^{*}$ is a canonical conjugate basis of $U^{*}, e_{i}^{*}\left(e_{j}\right)=\delta_{i j}$.

$T$ defines the barycentric coordinate map $\mathbb{T}: H \rightarrow V, \mathbb{T}(\alpha)=\sum_{i=1}^{4} p_{i} \otimes$ $e_{i}^{*}(\alpha)=\sum_{i=1}^{4} \alpha_{i} p_{i}$, where $\alpha=\sum_{i=1}^{4} \alpha_{i} e_{i} \in H$, and geometrically $T=\mathbb{T}(H \cap$ $\left.\left\{\alpha_{i} \geq 0\right\}\right)$. We see that any affine mapping $F: V \rightarrow V$ lifts to a linear transformation $M: U \rightarrow U$ of barycentric space preserving the hyperplane $H$, i.e. the following diagram commutes

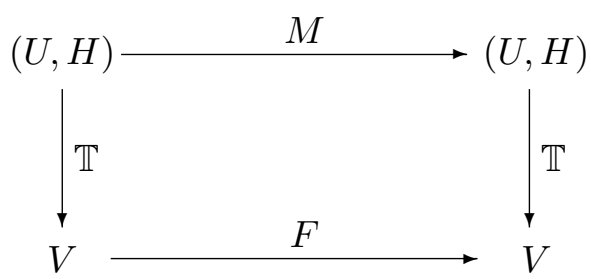

This diagram defines a barycentric representation of affine maps in $V$, where $M(H)=H$. To each $F$ we uniquely associate a linear homogeneous 
map in $U$ preserving $H$. If $T \in \mathcal{T}, T=\sum_{i=1}^{4} p_{i} \otimes e_{i}^{*}$, then its transformation by an affine mapping $F: V \rightarrow V, \bar{F}(T)=\sum_{i=1}^{4} F\left(p_{i}\right) \otimes e_{i}^{*} \in \mathcal{T}$ is represented by a map $M$ of barycentric space $(U, H)$ through the commuting diagram above,

$$
\mathbb{T}(M(\bullet))=F(\mathbb{T}(\bullet))
$$

and the affine action of $F$ onto $T$ can be expressed by a linear transformation $M$ preserving $H$ in the barycentric space. First we express uniquely $F\left(p_{i}\right)$ through $\left\{p_{1}, \ldots, p_{4}\right\}$ in barycentric coordinates $\alpha_{j i}$ in such a way that $F\left(p_{i}\right)=\sum_{j=1}^{4} \alpha_{j i} p_{j}, \sum_{j=1}^{4} \alpha_{j i}=1, i=1, \ldots, 4$. Then we write

$$
\begin{aligned}
F(\mathbb{T}(\alpha)) & =\sum_{i=1}^{4} F\left(p_{i}\right) \otimes e_{i}^{*}(\alpha)=\sum_{i=1}^{4} \sum_{j=1}^{4} \alpha_{j i} p_{j} \otimes e_{i}^{*}(\alpha) \\
& =\sum_{j=1}^{4} p_{j} \otimes \sum_{i=1}^{4} \alpha_{j i} e_{i}^{*}(\alpha)=\mathbb{T}(M(\alpha)) \\
& =\sum_{i=1}^{4} p_{i} \otimes e_{i}^{*}(M(\alpha))=\sum_{i=1}^{4} p_{i} \otimes M^{*}\left(e_{i}^{*}\right)(\alpha),
\end{aligned}
$$

where $M^{*}$ is a conjugate mapping and we write $M=\left(\alpha_{j i}\right)$.

Thus we have got the formula,

$$
\sum_{i=1}^{4} \sum_{j=1}^{4} \alpha_{j i} p_{j} \otimes e_{i}^{*}=\sum_{j=1}^{4} p_{j} \otimes\left(\sum_{i=1}^{4} \alpha_{j i} e_{i}^{*}\right)=\sum_{j=1}^{4} p_{j} \otimes M^{*}\left(e_{j}^{*}\right)
$$

An ordered sequence $\left\{T^{(i)}\right\}_{i=0}^{n}$ of regular tetrahedra is called a tetrahedral chain if any pair of two consecutive tetrahedra have a common face.

We denote by $s_{i}$ the center of $S_{i}, s_{i}=\frac{1}{3}\left(\sum_{j=1}^{4} p_{j}-p_{i}\right)$. Four reflections $R_{i}$ are defined uniquely by $S_{i}$ and their orthogonals $s_{i}-p_{i}, \quad 1 \leq i \leq 4$,

$$
R_{i}(p)=p-2 \frac{\left\langle p-s_{i}, s_{i}-p_{i}\right\rangle}{\left\langle s_{i}-p_{i}, s_{i}-p_{i}\right\rangle}\left(s_{i}-p_{i}\right),
$$

where $\langle.,$.$\rangle denotes the inner product.$

For a given tetrahedron $T$ the tetrahedral triangulation process is attaching a copy of $T$ to one of its faces. A natural step of the triangulation process is defined by reflection of the vertex $p_{i}$ in the face $S_{i}$. The new attached tetrahedron is defined by three vertices lying on the face $S_{i}$ and the reflected vertex $p_{i}^{(1)_{i}}$. We denote it by $T_{i}^{(1)}$ as it is uniquely determined 
by an extra vertex $p_{i}^{(1)_{i}}$ after one step-reflection of triangulation process. The new attached tetrahedron $T_{i}^{(1)}$ has four vertices

$$
\left\{p_{1}^{(1)_{i}}=R_{i}\left(p_{1}\right), p_{2}^{(1)_{i}}=R_{i}\left(p_{2}\right), p_{3}^{(1)_{i}}=R_{i}\left(p_{3}\right), p_{4}^{(1)_{i}}=R_{i}\left(p_{4}\right)\right\},
$$

where

$$
R_{i}\left(p_{j}\right)=p_{j}, \quad j \neq i
$$

as $p_{j}-s_{i}$ is orthogonal to $s_{i}-p_{i}$, and for $j=i$,

$$
R_{i}\left(p_{i}\right)=p_{i}+2\left(s_{i}-p_{i}\right)
$$

that is,

$$
R_{i}\left(p_{j}\right)=p_{j}^{(1)_{i}}=p_{j}+2 \delta_{i j}\left(\frac{1}{3} \sum_{k \neq i} p_{k}-p_{j}\right), \quad j=1, \ldots, 4
$$

We write $T_{i}^{(1)}=\bar{R}_{i} T$ where $\bar{R}_{i}$, acting in the space $\mathcal{T}$, is defined by reflection $R_{i}$ (see 2) and it is called a reflect-morphism of $\mathcal{T}, \bar{R}_{i}^{2}=i d$.

Tetrahedral chains of lengths $1,2, \ldots, n$ are encoded and described in affine coordinates by compositions of $\bar{R}_{i}, i=1, \ldots, 4$ :

$$
\begin{aligned}
T^{(0)} & =T \\
T_{i_{1}}^{(1)} & =\bar{R}_{i_{1}} T \\
T_{i_{1} i_{2}}^{(2)} & =\bar{R}_{i_{2}} \bar{R}_{i_{1}} T, \quad i_{1} \neq i_{2}, \\
& \ldots \quad \cdots \quad \cdots \\
T_{i_{1} i_{2} \cdots i_{n}}^{(n)} & =\bar{R}_{i_{n}} \cdots \bar{R}_{i_{2}} \bar{R}_{i_{1}} T, \quad i_{k} \neq i_{k+1}, k=1, \ldots, n-1 .
\end{aligned}
$$

The tetrahedral chain growth is defined by the reflect-morphisms $\bar{R}_{i}$ (reflections) defined in (4) which operate in the space of regular tetrahedra $\mathcal{T}$. Each reflect-morphism defines a linear mapping operating on its second component;

$$
\bar{R}_{i}: \mathcal{T} \rightarrow \mathcal{T}, \quad \bar{R}_{i}\left(v \otimes u^{*}\right)=v \otimes M_{i}^{*} u^{*}
$$

First we formulate the following useful 
Lemma 2.1. The reflect-morphism $\bar{R}_{i}$ providing the tetrahedron $T_{i}^{(1)}=$ $\bar{R}_{i} T$ by reflection of $p_{i}$ in the face $S_{i}$ is defined, by an operator $M_{i}$ acting on barycentric space $(U, H), M_{i}(H)=H$ by the following formula

$$
M_{i}^{*} e_{k}^{*}=e_{k}^{*}+\frac{2}{3}\left(1-4 \delta_{i k}\right) e_{i}^{*}, \quad M_{i}^{* 2}=i d,
$$

where $1 \leq i, k \leq 4$

Proof. We have

$$
\begin{aligned}
\bar{R}_{i} T & =\sum_{j=1}^{4} p_{j} \otimes M_{i}^{*} e_{j}^{*}=\sum_{j=1}^{4} p_{j} \otimes\left(e_{j}^{*}+\frac{2}{3}\left(1-4 \delta_{i j}\right) e_{i}^{*}\right) \\
& =\sum_{j=1}^{4} p_{j} \otimes e_{j}^{*}+\sum_{l=1}^{4} p_{l} \otimes \frac{2}{3}\left(1-4 \delta_{i l}\right) e_{i}^{*} \\
& =\sum_{j=1}^{4} p_{j} \otimes e_{j}^{*}+\left[\sum_{l=1}^{4} \frac{2}{3}\left(1-4 \delta_{i l}\right) p_{l}\right] \otimes e_{i}^{*} \\
& =\sum_{j=1}^{4} p_{j} \otimes e_{j}^{*}+\left(\frac{2}{3} \sum_{l=1}^{4} p_{l}-\frac{8}{3} p_{i}\right) \otimes e_{i}^{*} \\
& =\sum_{j=1}^{4} p_{j} \otimes e_{j}^{*}+\left(\frac{2}{3} \sum_{k \neq i} p_{k}-2 p_{i}\right) \otimes e_{i}^{*} \\
& =\sum_{j=1}^{4} p_{j} \otimes e_{j}^{*}+\sum_{j=1}^{4}\left[2 \delta_{i j}\left(\frac{1}{3} \sum_{k \neq i} p_{k}-p_{j}\right)\right] \otimes e_{j}^{*} \\
& =\sum_{j=1}^{4}\left(p_{j}+2 \delta_{i j}\left(\frac{1}{3} \sum_{k \neq i} p_{k}-p_{j}\right)\right) \otimes e_{j}^{*}=\sum_{j=1}^{4} p_{j}^{(1)_{i}} \otimes e_{j}^{*} .
\end{aligned}
$$

In local coordinates we have the following matrix form of $M_{i}$,

$$
M_{1}=\left(\begin{array}{cccc}
-1 & \frac{2}{3} & \frac{2}{3} & \frac{2}{3} \\
0 & 1 & 0 & 0 \\
0 & 0 & 1 & 0 \\
0 & 0 & 0 & 1
\end{array}\right)^{T}, \quad M_{2}=\left(\begin{array}{cccc}
1 & 0 & 0 & 0 \\
\frac{2}{3} & -1 & \frac{2}{3} & \frac{2}{3} \\
0 & 0 & 1 & 0 \\
0 & 0 & 0 & 1
\end{array}\right)^{T}
$$




$$
M_{3}=\left(\begin{array}{cccc}
1 & 0 & 0 & 0 \\
0 & 1 & 0 & 0 \\
\frac{2}{3} & \frac{2}{3} & -1 & \frac{2}{3} \\
0 & 0 & 0 & 1
\end{array}\right)^{T}, \quad M_{4}=\left(\begin{array}{cccc}
1 & 0 & 0 & 0 \\
0 & 1 & 0 & 0 \\
0 & 0 & 1 & 0 \\
\frac{2}{3} & \frac{2}{3} & \frac{2}{3} & -1
\end{array}\right)^{T}
$$

Using $M_{i}$ in barycentric space $U$ we can write the n-th tetrahedron in a chain by composition of barycentric coordinate maps,

$$
\mathbb{T}_{i_{1} \cdots i_{n}}^{(n)}=\mathbb{T} M_{i_{1}} \cdots M_{i_{n}}
$$

Acting on vertices $\left\{p_{1}, p_{2}, p_{3}, p_{4}\right\}, \bar{R}_{i}$ is represented by the transpose of $M_{i}$. As an example we show the matrix form of the $\bar{R}_{1}$ - action.

$$
\bar{R}_{1}\left(\sum_{i=1}^{4} p_{i} \otimes e_{i}^{*}\right)=\sum_{i=1}^{4} p_{i}^{(1)_{1}} \otimes e_{i}^{*}
$$

where

$$
\left(\begin{array}{l}
p_{1}^{(1)_{1}} \\
p_{2}^{(1)_{1}} \\
p_{3}^{(1)_{1}} \\
p_{4}^{(1)_{1}}
\end{array}\right)=\left(\begin{array}{cccc}
-1 & \frac{2}{3} & \frac{2}{3} & \frac{2}{3} \\
0 & 1 & 0 & 0 \\
0 & 0 & 1 & 0 \\
0 & 0 & 0 & 1
\end{array}\right)\left(\begin{array}{l}
p_{1} \\
p_{2} \\
p_{3} \\
p_{4}
\end{array}\right)
$$

then the composition of reflect-morphisms acting on the tetrahedra reads,

$$
T_{i_{1} \cdots i_{n}}^{(n)}=\bar{R}_{i_{n}} \cdots \bar{R}_{i_{1}} T
$$

\section{Coding in the triplets of consecutive steps}

The mapping prescribing an affine center point of a tetrahedron; $C: \mathcal{T} \rightarrow V$,

$$
C(T)=\mathbb{T}\left(\frac{1}{4} \sum_{l=1}^{4} e_{l}\right)=\sum_{i=1}^{4} p_{i} \otimes e_{i}^{*}\left(\frac{1}{4} \sum_{l=1}^{4} e_{l}\right)=\frac{1}{4} \sum_{i=1}^{4} p_{i}
$$

and the corresponding vectors joining centers of two consecutive tetrahedra in a chain are defined by

$$
\begin{aligned}
x_{r} & =C\left(T^{(r)}-T^{(r-1)}\right)=c^{(r)}-c^{(r-1)} \\
& =\left(\left(\bar{R}_{i_{r}}-I d\right) \bar{R}_{i_{r-1}} \cdots \bar{R}_{i_{1}} T\right)\left(\alpha_{c}\right), \quad 1 \leq r \leq n,
\end{aligned}
$$

where $c^{(r)}$ is the center of a tetrahedron $T^{(r)}$,

$$
c^{(r)}=C\left(T^{(r)}\right)=\mathbb{T}^{(r)}\left(\alpha_{c}\right), \quad n=0,1, \ldots,
$$


and $\alpha_{c}=\frac{1}{4} \sum_{l=1}^{4} e_{l}$. The skeleton of the tetrahedral chain is a broken line built from consecutive segments, intervals $\left|x_{r}\right|$ joining the consecutive centers (Figure 4),

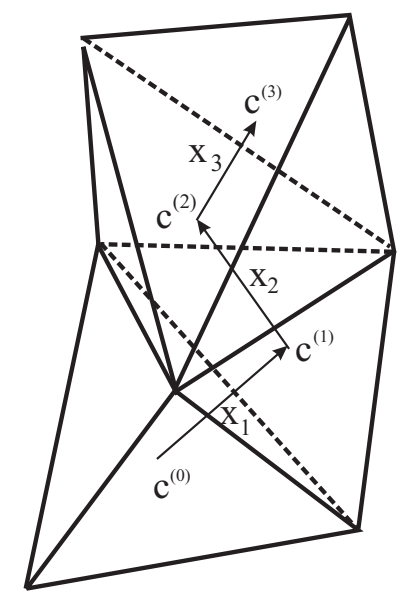

Figure 4: The orientation unit.

The three consecutive tetrahedra or two skeleton segments define an orientation plane spanned by two vectors $x_{r+2}$ and $x_{r+1}$. The corresponding tetrahedron $T_{i_{r+1} i_{r+2} i_{r+3}}^{(r+3)}=\bar{R}_{i_{r+3}} T_{i_{r+1} i_{r+2}}^{(r+2)}$ follow from the orientation tetrahedral unit, consisting of the three consecutive tetrahedra,

$$
T^{(r)}, \quad T_{i_{r+1}}^{(r+1)}=\bar{R}_{i_{r+1}} T^{(r)}, \quad T_{i_{r+1} i_{r+2}}^{(r+2)}=\bar{R}_{i_{r+2}} \bar{R}_{i_{r+1}} T^{(r)} .
$$

The three possible consecutive reflections $\bar{R}_{i_{r+1}}, \bar{R}_{i_{r+2}}, \bar{R}_{i_{r+3}}$ of an initial tetrahedron $T^{(r)}$ extend the initial tetrahedron to an oriented quadruple of four tetrahedra and determine the three skeleton segments $x_{r+1}, x_{r+2}, x_{r+3}$.

Definition 3.1. The three units $F, U, D$ of a tetrahedral chain, built from four consecutive tetrahedra are defined by three possible orientations of the three consecutive skeleton segments,

$$
\begin{array}{lll}
F: & T^{(r+3)} ; & \operatorname{det}\left(x_{r+1}, x_{r+2}, x_{r+3}\right)=0 \\
U: & T^{(r+3)} ; \quad \operatorname{det}\left(x_{r+1}, x_{r+2}, x_{r+3}\right)>0 \\
D: & T^{(r+3)} ; \quad \operatorname{det}\left(x_{r+1}, x_{r+2}, x_{r+3}\right)<0 .
\end{array}
$$


The initial value for continuation of the triangulation process, at each step of a process, is defined by $T^{(r)}$ with $r \geq 0$. A tetrahedral chain with fixed orientation in a space, i.e. with coordinates prescribed to each vertex of the chain, is written in the form of a word, e.g. $F \cdots F U \cdots U D \cdots D \cdots$.

The initial configuration of three consecutive tetrahedra $T^{(0)}, T_{i_{1}}^{(1)}, T_{i_{1} i_{2}}^{(2)}$, where $T^{(0)}=T$, establishes the exact position of a chain in $\mathbb{R}^{3}$. However the distribution of elements F,D,U along the word defines its geometric structure uniquely. In the symbolic description of a chain (invariant under $\mathrm{SO}(3)$ symmetries) we do not consider this initial configuration of the three consecutive tetrahedra and so we ignore its geometric position.

Proposition 3.2. At each element of a tetrahedral chain, say $T^{(r+3)}$, its position, $F, U, D$ is defined uniquely by the triplet of three consecutive tetrahedra $T^{(r)}, T^{(r+1)}, T^{(r+2)}$ and one of the three reflect-morphisms acting on $T^{(r+2)}$.

Proof. At each step of the triangulation process of a tetrahedral chain we have three preceding operations by reflect-morphisms,

$$
\begin{aligned}
& T_{k}^{(r+1)}=\bar{R}_{k} T^{(r)} \\
& T_{k j}^{(r+2)}=\bar{R}_{j} \bar{R}_{k} T^{(r)} \\
& T_{k j i}^{(r+3)}=\bar{R}_{i} \bar{R}_{j} \bar{R}_{k} T^{(r)} .
\end{aligned}
$$

Let $T^{(r)}=\sum_{l=1}^{4} p_{l} \otimes e_{l}^{*}$. Then we have,

$$
\begin{aligned}
T_{k}^{(r+1)} & =\bar{R}_{k} T^{(r)}=T^{(r)}+\frac{8}{3}\left(c^{(r)}-p_{k}\right) \otimes e_{k}^{*} \\
T_{k j}^{(r+2)} & =\bar{R}_{j} \bar{R}_{k} T^{(r)}=T^{(r+1)}+\frac{8}{3}\left(\frac{5}{3} c^{(r)}-p_{j}-\frac{2}{3} p_{k}\right) \otimes e_{j}^{*}, j \neq k \\
T_{k j i}^{(r+3)} & =\bar{R}_{i} \bar{R}_{j} \bar{R}_{k} T^{(r)} \\
& =T^{(r+2)}+\frac{8}{3}\left(\left(\frac{5}{3}+\beta_{i k}\right) c^{(r)}-p_{i}-\frac{2}{3} p_{j}-\beta_{i k} p_{k}\right) \otimes e_{i}^{*},
\end{aligned}
$$

for $\beta_{i k}=\frac{1}{9}\left(10-24 \delta_{i k}\right), i \neq j$

Now we distinguish separately the three configurations depending on the last reflect-morphism $\bar{R}_{i}$. Assuming $c^{(r)}=\frac{1}{4} \sum_{i=1}^{4} p_{i}=0$ we get, 


$$
\begin{gathered}
(F): i=k, x_{r+1}=-\frac{2}{3} p_{k}, x_{r+2}=-\frac{2}{3}\left(p_{j}+\frac{2}{3} p_{k}\right), x_{r+3}=-\frac{4}{9}\left(p_{j}+\frac{5}{6} p_{k}\right) \\
(U): i \neq k, x_{r+1}=-\frac{2}{3} p_{k}, x_{r+2}=-\frac{2}{3}\left(p_{j}+\frac{2}{3} p_{k}\right), \\
x_{r+3}=-\frac{2}{3}\left(p_{i}+\frac{2}{3} p_{j}+\frac{10}{9} p_{k}\right), \operatorname{det}\left(p_{k}, p_{j}, p_{i}\right)<0 \\
(D): i \neq k, x_{r+1}=-\frac{2}{3} p_{k}, x_{r+2}=-\frac{2}{3}\left(p_{j}+\frac{2}{3} p_{k}\right), \\
x_{r+3}=-\frac{2}{3}\left(p_{i}+\frac{2}{3} p_{j}+\frac{10}{9} p_{k}\right), \operatorname{det}\left(p_{k}, p_{j}, p_{i}\right)>0 .
\end{gathered}
$$

These configurations uniquely determine the three possible elements of the tetrahedral chain.

Definition 3.3. A triplet of integers $(k, j, i), 1 \leq i, j, k \leq 4$ such that $k \neq$ $j \neq i$ is called an admissible triplet. The set of all admissible triplets we denote by $A$.

By Proposition 3.2, to each admissible triplet of integers encoding one of the $F, U, D$ elements of a chain we associate a corresponding triplet of reflect-morphisms,

$$
(k, j, i) \mapsto\left(\bar{R}_{k}, \bar{R}_{j}, \bar{R}_{i}\right)
$$

As it is seen from the proof of Proposition 3.2 to complete orientation in the directed chain of tetrahedra and define especially the $U$ and $D$ chain elements we need to orient at least one tetrahedron of the chain. This means that if we fix the sign, say $\operatorname{det}\left[p_{1}, p_{2}, p_{3}\right]>0$, then the orientation is defined for all other admissible triplets. In what follows we will assume this orientation for the initial tetrahedron. By straightforward calculation (for standard tetrahedron inscribed in the unit sphere) based on the proof of Proposition 3.2 we get the following result,

Proposition 3.4. Assume that the normalization conditions, $\sum_{i=1}^{4} p_{i}=0$, and $\operatorname{det}\left(p_{1}, p_{2}, p_{3}\right)>0$, are satisfied. Then the classes of admissible triplets representing the first step $u, d$, and $f$ chain oriented elements are displayed in Table 1 . 


\begin{tabular}{|c|c|c|}
\hline$d$ & $f$ & $u$ \\
\hline $\operatorname{det}\left(x_{1}, x_{2}, x_{3}\right)=-32 \sqrt{3} / 243$ & $\operatorname{det}\left(x_{1}, x_{2}, x_{3}\right)=0$ & $\operatorname{det}\left(x_{1}, x_{2}, x_{3}\right)=32 \sqrt{3} / 243$ \\
\hline$(k, j, i)$ & $(k, j, i)$ & $(k, j, i)$ \\
\hline$(1,2,3)$ & $(3,2,3)$ & $(3,2,1)$ \\
\hline$(1,3,4)$ & $(4,3,4)$ & $(4,3,1)$ \\
\hline$(1,4,2)$ & $(4,2,4)$ & $(4,4,1)$ \\
\hline$(2,1,4)$ & $(1,2,1)$ & $(1,3,2)$ \\
\hline$(2,3,1)$ & $(4,1,4)$ & $(3,4,2)$ \\
\hline$(2,4,3)$ & $(3,1,3)$ & $(2,1,3)$ \\
\hline$(3,1,2)$ & $(3,4,3)$ & $(2,3,4)$ \\
\hline$(3,2,4)$ & $(2,1,2)$ & $(1,4,3)$ \\
\hline$(3,4,1)$ & $(1,3,1)$ & $(3,1,4)$ \\
\hline$(4,1,3)$ & $(1,4,1)$ & $(1,2,4)$ \\
\hline$(4,2,1)$ & $(2,4,2)$ & $(4,2,3)$ \\
\hline$(4,3,2)$ & $(2,3,2)$ & \\
\hline
\end{tabular}

Table 1: Classification of admissible triplets.

Each tetrahedron of a tetrahedral chain is given by reflection of the previous one. At each step of determining of the $D, F, U$ positions we have to normalize the initial conditions (initial tetrahedron) as it is described in Proposition 3.4, and follow the part of the proof of Proposition 3.2. Indeed if the initial tetrahedron, say $T^{(r)}$, was oriented by $\operatorname{det}\left[p_{1}, p_{2}, p_{3}\right]>0$, then the next one $T^{(r+1)}$, serving as an initial for the next step of determination of the $D, F, U$ elements, is given as a reflection of a previous one, so after normalization of its coordinates, $\sum_{i=1}^{4} p_{i}=0$ we get the opposite orientation of the initial condition, i.e. $\operatorname{det}\left[p_{1}, p_{2}, p_{3}\right]<0$. And this orientation changes at each even step of our construction. Thus choosing an admissible triplet, say for $D$ in the second step we choose it from the $u$ column of admissible triplets of Table 1. In the next step (odd) of this procedure the initial data orientation comes to the first one, i.e. $\operatorname{det}\left[p_{1}, p_{2}, p_{3}\right]>0$, and we use the Table 1 as it is. This is the reason why we denoted the admissible triplets by lower case characters $f, u, d$. And $f$ corresponds to the geometric form $F$ along the chain, but $u$ and $d$ interchange $D$ and $U$ along the chain depending on orientation of the local initial data.

To avoid this varying initial orientation data along the triangulation process we introduce, using duality concept defined below, the notion of an adjoint word in the lower case characters of admissible triplets. 
Definition 3.5. To each tetrahedral chain written in the form of word $W$ (possibly infinite), for example

$$
W=U U D D U U F F U \cdots
$$

we associate uniquely a new word $\bar{W}=I(W)$ which is called an adjoint word. The adjoint word is defined by replacing each character $U$ (or $D$ ) by $d$ (or $u$ respectively) if it is in an even position of the word $W$. All $F$ characters are replaced by $f$ in its adjoint form, for example (resp.)

$$
I(W)=\bar{W}=u d d u u d f f u \cdots .
$$

$I$ is a duality between the spaces of admissible words, one written in the capital characters and the another one written in the lower case characters.

Reducing the word characters along the chain to the ones listed in Table 1 which are the first step characters represented numerically we show that each tetrahedral chain can be uniquely represented by a sequence of admissible triplets of integers. This sequence of triplets is defined by an initial triplet and the triplets of Table 1 .

\section{Numerical invariants of the tetrahedral chains}

Any tetrahedral chain is described as a composition of admissible ordered triplets representing the corresponding characters $D, F, U$ of a defining word, e.g. for $U U D F D$ we have $I(U U D F D)=u d d f d$ and respectively,

$$
(3,4,2) \rightarrow(4,2,1) \rightarrow(2,1,4) \rightarrow(1,4,1) \rightarrow(4,1,3) .
$$

and

$$
T_{3421413}^{(7)}=\bar{R}_{3} \bar{R}_{1} \bar{R}_{4} \bar{R}_{1} \bar{R}_{2} \bar{R}_{4} \bar{R}_{3} T .
$$

Each admissible triplet $\left(i_{1}, i_{2}, i_{3}\right)$ is a mapping from an ordered set $\Delta_{3}=\{1,2,3\}$ to the set $\Delta_{4}=\{1,2,3,4\}$ denoted by $f_{i_{1} i_{2} i_{3}}, f_{i_{1} i_{2} i_{3}}(j)=i_{j} \in$ $\Delta_{4}, j=1,2,3$. There is a natural action of the direct product of symmetric groups $S_{4} \times S_{3}$ in the space of such mappings,

$$
(\xi, \sigma) . f_{i_{1} i_{2} i_{3}}=\xi \circ f_{i_{1} i_{2} i_{3}} \circ \sigma, \quad \xi \in S_{4}, \sigma \in S_{3}
$$

The set of all mappings $f$ into admissible triplets, i.e. $f_{i_{1} i_{2} i_{3}}\left(\Delta_{3}\right) \in A$ we denote by $\mathcal{A}$. Obviously $\mathcal{A}$ is not preserved by the above defined group action. 
Now we fix an orientation of a chain choosing a reference triplet $(i, j, k)$, $i \neq j \neq k \neq i$. We call this triplet positive. One can easily see that any mapping $f_{i_{1} i_{2} i_{3}} \in \mathcal{A}$ can be reconstructed from the reference mapping $f_{i j k}$ by the group action, i.e.

$$
f_{i_{1} i_{2} i_{3}}=\xi \circ f_{i j k} \circ \sigma
$$

Lemma 4.1. 1. If $i_{1} \neq i_{2} \neq i_{3} \neq i_{1}, f_{i_{1} i_{2} i_{3}}(s) \in\{i, j, k\}, 1 \leq s \leq 3$, then there is a uniquely defined $\sigma \in S_{3}$ such that $f_{i_{1} i_{2} i_{3}}=(i d, \sigma) . f_{i j k}$.

2. If $i_{1} \neq i_{2} \neq i_{3} \neq i_{1},\left\{i_{1}, i_{2}, i_{3}\right\} \neq\{i, j, k\}$, then there are uniquely defined, transposition $\tau \in S_{4}$ and permutation $\sigma \in S_{3}$ such that $f_{i_{1} i_{2} i_{3}}=$ $(\tau, \sigma) . f_{i j k}$.

Proof. The case 1.) is straightforward as an image set of $f_{i_{1} i_{2} i_{3}}$ is equal to $\{i, j, k\}$. In the case 2.) there are three possibilities $\left\{i_{1}, i_{2}, i_{3}\right\}=\{i, j, l\}$, $\left\{i_{1}, i_{2}, i_{3}\right\}=\{i, l, k\}$, and $\left\{i_{1}, i_{2}, i_{3}\right\}=\{l, j, k\}$, where $\{l\}=\Delta_{4}-\{i, j, k\}$. In each of these cases there is one of the transpositions $\tau_{k l}, \tau_{j l}, \tau_{i l}$ such that the image of $f_{i_{1} i_{2} i_{3}}$ is equal to $\tau_{*} \circ f_{i j k}$, where $\tau_{*}$ denotes one of the permutations $\tau_{k l}, \quad \tau_{j l}$ and $\tau_{i l}$. Then there is a uniquely defined element $\sigma \in S_{3}$ such that $f_{i_{1} i_{2} i_{3}}=\tau_{*} \circ f_{i j k} \circ \sigma$.

On the basis of Lemma 4.1 and straightforward checking, we get a new numerical characteristic of admissible triplets.

Proposition 4.2. To each admissible triplet $f_{i_{1} i_{2} i_{3}}$ we associate uniquely its sign $\Lambda$, i.e. there is a mapping $\Lambda: \mathcal{A} \rightarrow\{-1,0,+1\}$, such that

1. $\Lambda\left(f_{i j k}\right)=+1$

2. If $i_{1} \neq i_{2} \neq i_{3} \neq i_{1}, \quad f_{i_{1} i_{2} i_{3}}(s) \in\{i, j, k\}, \quad 1 \leq s \leq 3$, then $f_{i_{1} i_{2} i_{3}}=$ $(i d, \sigma) . f_{i j k}$ and $\Lambda\left(f_{i_{1} i_{2} i_{3}}\right)=\operatorname{sgn}(\sigma)$.

3. If $i_{1} \neq i_{2} \neq i_{3} \neq i_{1},\left\{i_{1}, i_{2}, i_{3}\right\} \neq\{i, j, k\}$, then $f_{i_{1} i_{2} i_{3}}=\left(\tau_{*}, \sigma\right)$. $f_{i j k}$ and $\Lambda\left(f_{i_{1} i_{2} i_{3}}\right)=\operatorname{sgn}\left(\tau_{*}\right) \operatorname{sgn}(\sigma)=-\operatorname{sgn}(\sigma)$.

4. If $i_{1}=i_{3}, \Lambda\left(f_{i_{1} i_{2} i_{3}}\right)=0$.

As a formal notion for $\Lambda$ we propose its representation as the sign of the determinant built by the three vertices of the initial tetrahedron of the tetrahedral chain, i.e. the positive reference triplet means $\operatorname{det}\left(p_{i}, p_{j}, p_{k}\right)>0$ 
$\left(\operatorname{sgn}\left(\operatorname{det}\left(p_{i}, p_{j}, p_{k}\right)\right)=+1\right)$, and

$$
\Lambda\left(f_{i_{1} i_{2} i_{3}}\right)=\operatorname{sgn}\left(\operatorname{det}\left(p_{i_{1}}, p_{i_{2}}, p_{i_{3}}\right)\right)
$$

Now we investigate the properties of $\Lambda$ represented by this determinant and stated in Proposition 4.2. We show that

$$
\begin{aligned}
\Lambda\left(f_{i_{1} i_{2} i_{3}}\right) & =\operatorname{sgn}\left(\operatorname{det}\left(p_{i_{1}}, p_{i_{2}}, p_{i_{3}}\right)\right)=\Lambda\left(\xi \circ f_{i j k} \circ \sigma\right) \\
& =-\operatorname{sgn}(\sigma) \operatorname{sgn}\left(\operatorname{det}\left(p_{i}, p_{j}, p_{k}\right)\right) .
\end{aligned}
$$

Indeed,

$$
\begin{aligned}
& \operatorname{det}\left(p_{\xi \circ f_{i j k} \circ \sigma(1)}, p_{\xi \circ f_{i j k} \circ \sigma(2)}, p_{\xi \circ f_{i j k} \circ \sigma(3)}\right) \\
= & \operatorname{sgn}(\sigma) \operatorname{det}\left(p_{\xi \circ f_{i j k}(1)}, p_{\xi \circ f_{i j k}(2)}, p_{\xi \circ f_{i j k}(3)}\right) \\
= & \operatorname{sgn}(\sigma) \operatorname{det}\left(p_{\xi(i)}, p_{\xi(j)}, p_{\xi(k)}\right)=\operatorname{sgn}(\sigma) \operatorname{det}\left(p_{i}, p_{j}, p_{l}\right),
\end{aligned}
$$

because we choose (as an example) $\xi=\tau_{k l}$. And taking $p_{l}=-\left(p_{i}+p_{j}+p_{k}\right)$ we get

$$
\begin{aligned}
\operatorname{sgn}(\sigma) \operatorname{det}\left(p_{i}, p_{j}, p_{l}\right) & =\operatorname{sgn}(\sigma) \operatorname{det}\left(p_{i}, p_{j},-p_{i}-p_{j}-p_{k}\right) \\
& =-\operatorname{sgn}(\sigma) \operatorname{det}\left(p_{i}, p_{j}, p_{k}\right) .
\end{aligned}
$$

In the sequence of admissible triplets defining a tetrahedral chain for a given triplet of the sequence the two last numbers of this triplet repeat in the subsequent triplet in the same order. To each tetrahedral chain $W_{n}$ we associate the chain of admissible triplets.

Definition 4.3. The chain of admissible triplets

$$
\cdots \stackrel{\tilde{L}_{*}}{\longrightarrow}\left(i_{1}, i_{2}, i_{3}\right) \stackrel{\tilde{L}_{*}}{\longrightarrow}\left(i_{2}, i_{3}, i_{4}\right) \stackrel{\tilde{L}_{*}}{\longrightarrow}\left(i_{3}, i_{4}, i_{5}\right) \stackrel{\tilde{L}_{*}}{\longrightarrow} \cdots
$$

where $\tilde{L}_{*}$ is defined by the corresponding transposition $\tau_{*} \in S_{4}$, maximal order element (maximal cycle) $\gamma$ of $S_{3}$ and the unique relation

$$
f_{i_{r+2} i_{r+3} i_{r+4}}=\tau_{*} \circ f_{i_{r+1} i_{r+2} i_{r+3}} \circ \gamma
$$

is called an admissible chain and we denote it by $Q_{n}$.

Action of the above defined pairs $\left(\tau_{*}, \gamma\right) \in S_{4} \times S_{3}$ preserves the space of admissible mappings $\mathcal{A}$. The relation of sign of $\Lambda$ in an admissible sequence 
reads

$$
\Lambda\left(f_{i_{2} i_{3} i_{4}}\right)=\Lambda\left(\tau_{*} \circ f_{i_{1} i_{2} i_{3}} \circ \gamma\right)=-\operatorname{sgn}(\gamma) \Lambda\left(f_{i_{1} i_{2} i_{3}}\right) .
$$

In the space of all finite admissible chains of triplets we introduce the following equivalence,

Definition 4.4. Two admissible chains $Q_{n}$ and $Q_{n}^{\prime}$

$$
\begin{aligned}
Q_{n}=\cdots \stackrel{\tilde{L}_{*}}{\longrightarrow}\left(i_{r+1}, i_{r+2}, i_{r+3}\right) & \stackrel{\tilde{L}_{*}}{\longrightarrow}\left(i_{r+2}, i_{r+3}, i_{r+4}\right) \\
& \stackrel{\tilde{L}_{*}}{\longrightarrow}\left(i_{r+3}, i_{r+4}, i_{r+5}\right) \stackrel{\tilde{L}_{*}}{\longrightarrow} \cdots, \\
Q_{n}^{\prime}=\cdots \stackrel{\tilde{L}_{*}}{\longrightarrow}\left(j_{r+1}, j_{r+2}, j_{r+3}\right) & \stackrel{\tilde{L}_{*}}{\longrightarrow}\left(j_{r+2}, j_{r+3}, j_{r+4}\right) \\
& \stackrel{\tilde{L}_{*}}{\longrightarrow}\left(j_{r+3}, j_{r+4}, j_{r+5}\right) \stackrel{\tilde{L}_{*}}{\longrightarrow} \cdots,
\end{aligned}
$$

are equivalent, $Q_{n} \sim Q_{n}^{\prime}$, if there exists $\xi \in S_{4}$ such that $f_{i_{k+1} i_{k+2} i_{k+3}}=\xi \circ$ $f_{j_{k+1} j_{k+2} j_{k+3}}$ for $k=0, \ldots, n-1$.

To each admissible chain, say $Q_{n}$, we associate its sign sequence

$$
\Lambda\left(Q_{n}\right)=\left\{\Lambda\left(f_{i_{1} i_{2} i_{3}}\right), \ldots, \Lambda\left(f_{i_{n} i_{n+1} i_{n+2}}\right)\right\} .
$$

Proposition 4.5. The sign sequence is a numerical invariant, i.e.

$$
\Lambda\left(Q_{n}\right)=\Lambda\left(Q_{n}^{\prime}\right) \text { if and only if } Q_{n} \sim Q_{n}^{\prime}
$$

Thus the sign sequences corresponding to the admissible sequences are in 1-1 correspondence with the tetrahedral chains. There is a natural correspondence of these two sequences,

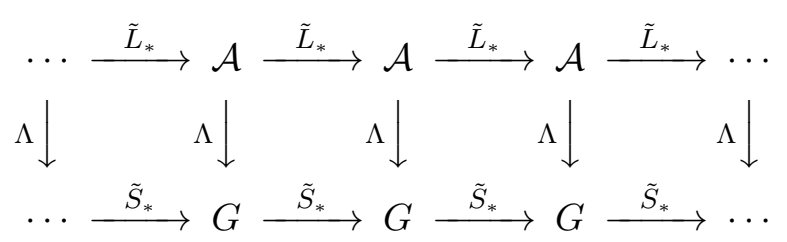
to $\tilde{L}_{*}$.

where $G=\{-1,0,+1\}$ and $\tilde{S}_{*}$ is an operator $G \rightarrow G$ corresponding

\section{Geometric characteristics}

All finite regular tetrahedral chains or equivalently the finite words consisting of $D, F, U$ or by duality $u, d, f$ characters are denoted by $\Gamma_{\mathcal{T}}$. It is a 
collection of chains $C_{n} \in \Gamma_{\mathcal{T}}(n)$ of length $n, n \in \mathbb{N}, \Gamma_{\mathcal{T}}=\bigcup_{n \in \mathbb{N}} \Gamma_{\mathcal{T}}(n)$. All chains which do not self-intersect form a subspace of $\Gamma_{\mathcal{T}}$. We call them proper tetrahedral chains and denote by $\mathcal{C}_{\mathcal{T}}, \mathcal{C}_{\mathcal{T}}=\bigcup_{n \in \mathbb{N}} \mathcal{C}_{\mathcal{T}}(n) \subset \Gamma_{\mathcal{T}}$, where $\mathcal{C}_{\mathcal{T}}(n)$ are proper tetrahedral chains of length $n$.

One of the geometric properties of tetrahedral chains is their clustering characteristic. First we introduce the notion of vertex order. If $p$ is a common vertex for a number of tetrahedra we call this number an order of vertex $p$. Vertex order is a function $P: V_{C_{n}} \rightarrow \mathbb{N}$ defined on an ordered sequence of vertices $V_{C_{n}}$ of chain $C_{n}$ which to each vertex $p \in V_{C_{n}}$ along the chain $C_{n}$ associate its order $P(p)$. In the same way we define an edge order function $G$ : $E_{C_{n}} \rightarrow \mathbb{N}$ defined on an ordered sequence of edges $E_{C_{n}} . G(l)$ is the number of tetrahedra which share an edge $l, l \in E_{C_{n}}$. We can easily check the following

Lemma 5.1. 1. The total vertex order $\tilde{V}=\sum_{p \in V_{C_{n}}} P(p)$ for a chain does not depend on a form of the chain and for the chain of length $n$ it is equal to $4 n$.

2. A maximal vertex order of the proper tetrahedral chain is 20 and all chains of length 20 with such vertex of maximal order are called icosi-clusters (icosihedral clusters). The distribution of values of the vertex order function along the chain defines uniquely up to orientation the geometric shape of the chain, i.e. if two functions $P_{1}$ and $P_{2}$ associated to two chains of the same length are equal then their shapes are identical up to orientation.

Remark 5.2. If the vertex order function along a chain is less than or equal to 4 , then the chain is a tetrahelix with $U$ or $D$ orientation. In this case the level sets of an edge order function $G, G^{-1}(1), G^{-1}(2), G^{-1}(3)$ form the three helices built from external edges, two broken helices made of edges of order two and the central broken spiral.

On each space $\mathcal{C}_{\mathcal{T}}(n) \subset \Gamma_{\mathcal{T}}(n)$ of the proper tetrahedral chains of length $n$, we have the clustering function $C l: \mathcal{C}_{\mathcal{T}}(n) \rightarrow \mathbb{N}$,

$$
C l\left(C_{n}\right)=\sum_{p \in V_{C_{n}}} \max (0, P(p)-4) .
$$

We see that the clustering function vanishes on the tetrahelix and has a maximal value for the chains consisting of a number of icosi-clusters, say $m$, and completed by a $k$-cluster (the chain composed of one common vertex of order $k)$ with $k=n(\bmod 20 m), k<20$.

For small $n$ we can classify all proper tetrahedral chains of length $n$. Up to $n=7$ a selfintersection appears only for $F F F$ part of the chain. If 
$8 \leq n \leq 10$ the selfintersected chains appear in chains with one common vertex, i.e. if all tetrahedra of a chain have a common vertex. The cases $n \geq 11$ add to the above possibilities the chains which are selfintersecting in long distance geometric structures.

Proposition 5.3. The numbers of proper chains $A_{n}$ of length $n \leq 13$ are listed in the following table.

\begin{tabular}{|c||c|c|c|c|c|c|c|c|c|c|c|}
\hline$n$ & 3 & 4 & 5 & 6 & 7 & 8 & 9 & 10 & 11 & 12 & 13 \\
\hline \hline$A_{n}$ & 1 & 3 & 9 & 26 & 76 & 218 & 628 & 1802 & 5146 & 14670 & 41734 \\
\hline
\end{tabular}

Table 2: Proper tetrahedral chains.

In the tetrahedral triangulation process of a proper chain at each final tetrahedron one may have at most three possibilities to attach the next tetrahedron. If we replace tetrahedrons by the equal tightly packed spheres, then the process of attaching the new tetrahedron corresponds to gluing an appropriate sphere and use the computational methods (cf. [5]). Thus we see that every tetrahedron along a chain has its branching order which is the number $b, \quad 0 \leq b \leq 3$ of possible concurrent continuations of the chain at the given tetrahedron. The zero branching order terminates the chain, i.e. each of the three attached tetrahedra intersect the chain. An example of zero branching order chain written in the form UUFFDDUUFFDDUFFDDDUDUU$D D U D D D F D D D U U U U F D U U U U U U F F D D D D D$, is shown in Figure 5. The branching order of the final tetrahedron in a chain is called the branching order of the chain. If the branching order of a chain is one, then at its final tetrahedron there is only one possibility of continuation of the chain.

Such chains with branching order one, form the basic units with substantially reduced space of possible configurations. Classification of proper chains with all tetrahedra having a fixed common vertex and fixed branching order is given in the following,

Theorem 5.1. The proper tetrahedral chains with all tetrahedra sharing one fixed geometrical vertex, with different branching orders $b=1$, 2 and 3 are listed in Table 3 . 


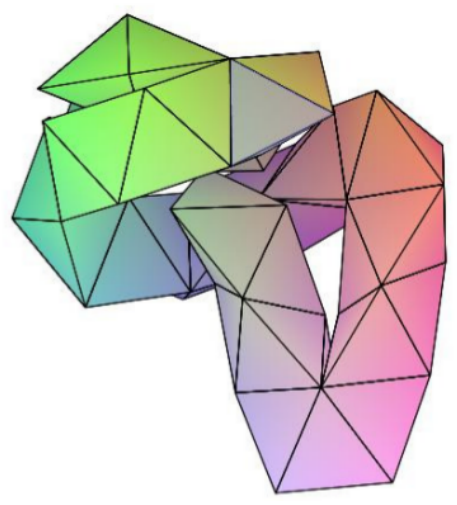

Figure 5: Example of chain with zero branching order

\begin{tabular}{|c||c|c|c|c|c|c|c|c|c|c|c|c|c|c|c|c|c||c|}
\hline$b \backslash n$ & 4 & 5 & 6 & 7 & 8 & 9 & 10 & 11 & 12 & 13 & 14 & 15 & 16 & 17 & 18 & 19 & 20 & total \\
\hline \hline 1 & 0 & 0 & 0 & 0 & 0 & 1 & 2 & 6 & 9 & 19 & 38 & 49 & 69 & 79 & 71 & 34 & 6 & 383 \\
\hline 2 & 0 & 0 & 1 & 4 & 6 & 10 & 24 & 46 & 78 & 113 & 137 & 153 & 132 & 85 & 36 & 6 & 0 & 831 \\
\hline 3 & 2 & 4 & 6 & 9 & 16 & 27 & 38 & 48 & 55 & 56 & 50 & 35 & 22 & 12 & 2 & 0 & 0 & 380 \\
\hline \hline total & 2 & 4 & 7 & 13 & 22 & 38 & 64 & 100 & 142 & 188 & 225 & 237 & 223 & 176 & 109 & 40 & 6 & 1594 \\
\hline
\end{tabular}

Table 3: Table of chains sharing one common vertex.

Example 5.4. The first listing of short proper chains centered at one fixed vertex with $4 \leq n \leq 8$ reads as follows:

$n=4: F, D$

$n=5: F F, F D, D U, D F$,

$n=6: F F D, F D F, F D U, D U F, D U D, D F F, D F U$

$n=7: F F D F, F F D U, F D F F, F D F U, F D U F, F D U D, D U F F$, $D U F D, D U D F, D U D U, D F F U, D F U F, D F U D$

$n=8: F F D F U, F F D F F, F F D U D, F D F F U, F D F U D, F D F U F$, FDUFD FDUDU, FDUDF, DUFFD, DUFDU, DUFDF, DUDFU, DUDFF DUDUD, DUDUF, DFFUF, DFFUD, DFUFD, DFUFF, DFUDU, DFUDF. 
Remark 5.5. 1. The shortest proper tetrahedral chain (cluster) centered at one fixed vertex of branching order $b=1, n=9$, is given by the code $U D F U D F$.

2. The icosi-clusters are listed in the following 6 codes (examples see Figure 60:

\section{FFUFFDUDUDFFUFFDU, FFUFFDUDUDUDFFUFF UFF DFFUDUDUFFDFFU, UFFDUDFFUFFDUDFFU UDFFUFFDUDFFUFFDU, UDFFUFFDUDUDFFUFF.}

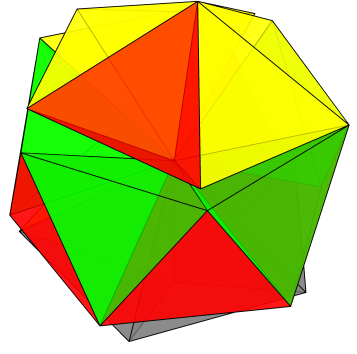

FFUFFDUDUDUDFFUFF

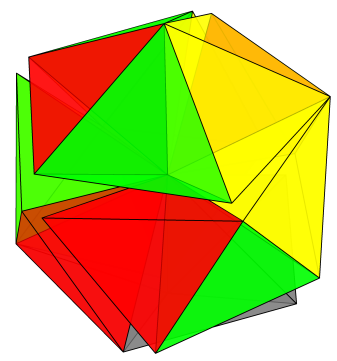

UDFFUFFDUDFFUFFDU

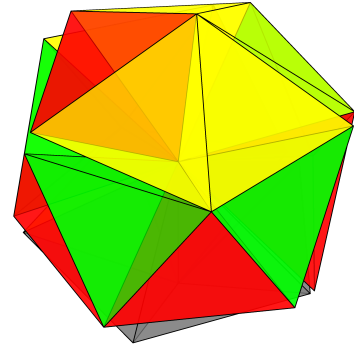

FFUFFDUDUDFFUFFDU

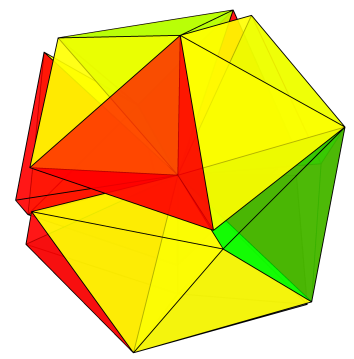

UFFDUDFFUFFDUDFFU

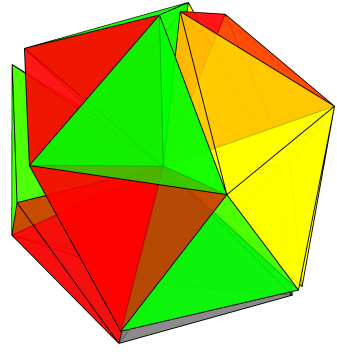

UDFFUFFDUDUDFFUFF

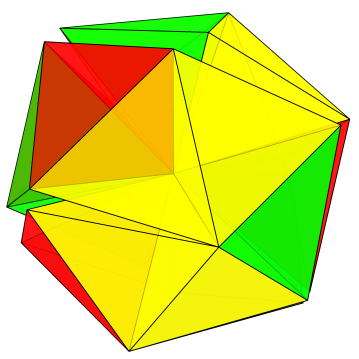

UFFDFFUDUDUFFDFFU

Figure 6: Six different configurations of icosi-clusters $n=20, b=1$.

3. Table 3 displays numbers of all proper tetrahedral chains whose tetrahedra share one geometrically fixed point as a vertex. This way we get part of the clusters, namely those which have a fixed orientation. Table 3 extended by numbers of all chains having codes with $U$ replaced by $D$ and vice versa completes the set of all proper tetrahedral chains with one common vertex. 


\section{Periodic tetrahedral chains}

A tetrahedral chain is called periodic if the configuration of elements $F, U, D$ in the defining word, $D \cdots D U \cdots U F \cdots$ is periodic. Any periodic tetrahedral chain is uniquely defined by its period which forms the shortest finite tetrahedral chain written in the form of word, say $W_{m}=U D F U \cdots D U$, of length $m$. We will call this word the basic period of a periodic tetrahedral chain.

In the reflect-morphism representation of a chain for each of $D, F, U$ unit along the chain we need two preceding reflect-morphisms which define an admissible triplet of integers. Thus the period in a numerical characterization of a periodic tetrahedral chain by its sequence of admissible triplets corresponds to the shortest cycle of the triplets which continues repeating along a sequence. The cycle of triplets is directly represented by reflectmorphisms and finally by their composition. This cycle forms a principal operator which is a $4 \times 4$ matrix for a periodic chain. In this section we show the existence of such a matrix for a periodic chain and display some of its properties.

To construct a coordinate representation of a periodic chain, i.e. the reflect-morphism composition for a cycle of admissible triplets, we define the corresponding adjoint defining sequence of a periodic tetrahedral chain and its adjoint period.

We observe that the shortest word $W_{m}$ is a period of a tetrahedral chain $W$ if its adjoint word $\bar{W}_{m}$ is also period for an adjoint defining sequence $\bar{W}$. We notice that if the length of a basic period $W_{m}$ is even, then the adjoint word $\bar{W}_{m}=I\left(W_{m}\right)$ is also a basic period for an adjoint defining sequence. Obviously the doubled basic period is a period for the adjoint defining sequence in the case of odd length of the basic period.

Let us assume the positive orientation of an initial tetrahedron $T=$ $\left\{p_{1}, p_{2}, p_{3}, p_{4}\right\}$, i.e., $\operatorname{det}\left[p_{1}, p_{2}, p_{3}\right]>0$, then using Table 1 for any two indices $i_{1}, i_{2}, \quad i_{1} \neq i_{2}, 1 \leq i_{1}, i_{2} \leq 4$ we associate uniquely an integer $i_{3}, 1 \leq i_{3} \leq$ 4 such that $\left(i_{1}, i_{2}, i_{3}\right)$ is an admissible triplet of the fixed type " $d, f, u^{\prime \prime}$. Thus we have defined the natural mappings $L_{u}, L_{d}, L_{f}: \mathbb{I} \rightarrow \Delta_{4}$, where $\Delta_{4}=$ $\{1,2,3,4\}$ and $\mathbb{I}=\left\{(\alpha, \beta) \in \Delta_{4} \times \Delta_{4}: \alpha \neq \beta\right\}$.

Any word $W_{m}$ of length $m$ can be represented as a composition of $m+2$ reflect-morphisms $\bar{R}_{i_{m+2}} \cdots \bar{R}_{i_{1}}$. A sequence of indices $i_{1}, \ldots, i_{m+2}$ is defined by the sequence of $L_{*}$-mappings (i.e. $L_{u}, L_{d}$ or $L_{f}$ ) directly corresponding to the sequence of characters in the dual word $\bar{W}_{m}$. 
Example 6.1. As an example the $L_{*}$ - sequence for $W_{m}=U D F U \cdots D U$ and $\bar{W}_{m}=u u f d \cdots d d$ reads,

$$
\begin{aligned}
L_{u}\left(i_{1}, i_{2}\right) & =i_{3}, \\
L_{u}\left(i_{2}, i_{3}\right) & =i_{4}, \\
L_{f}\left(i_{3}, i_{4}\right) & =i_{5}, \\
L_{d}\left(i_{4}, i_{5}\right) & =i_{6}, \\
\cdots & \cdots \\
L_{d}\left(i_{m-1}, i_{m}\right) & =i_{m+1}, \\
L_{d}\left(i_{m}, i_{m+1}\right) & =i_{m+2} .
\end{aligned}
$$

For any pair of initial integers $\left(i_{1}, i_{2}\right)$ from $\mathbb{I}$ the remaining indices $i_{k}, k=$ $3, \ldots, m+2$ are uniquely defined by Table 1 .

Taking the reflect-morphisms representation of a periodic tetrahedral chain with $W_{m}$ to be a period we see that the corresponding composition $\bar{R}_{i_{m+2}} \cdots \bar{R}_{i_{1}}$ does not necessarily continue periodically along a chain. It would be so if the $L_{*}$-sequence, i.e. ordered (according to $\bar{W}_{m}$ ) collection of mappings $L_{u}, L_{d}, L_{f}$ is cyclic, i.e., if $i_{1}=i_{m+1}, i_{2}=i_{m+2}$.

Example 6.2. This fact may be easily seen for the three basic periodic chains,

$$
\begin{aligned}
& U \text { periodic }: U \cdots U \cdots, \\
& D \text { periodic }: D \cdots D \cdots, \\
& F \text { periodic }: F \cdots F \cdots
\end{aligned}
$$

In the first two cases the period is a word of two characters, $U U, D D$. In $U$ (and $D$ ) periodic chains the defining numerical sequences are cyclic after four iterations of the $L_{*}$-sequence, $L_{u} L_{d}, L_{u} L_{d}$,

$$
\begin{array}{ll}
L_{u}\left(i_{1}, i_{2}\right)=i_{3}, & L_{d}\left(i_{2}, i_{3}\right)=i_{4}, \\
L_{u}\left(i_{3}, i_{4}\right)=i_{1}, & L_{d}\left(i_{4}, i_{1}\right)=i_{2}, \quad\left(i_{1}, i_{2}\right) \in \mathbb{I},
\end{array}
$$

which reads in numerical characters

$$
I(U U U U U U \cdots)=\operatorname{ududud} \cdots, \quad I(D D D D D D \cdots)=d u d u d u \cdots
$$

as displayed in Table 4. Obviously the $L_{*}$-sequence $L_{f} L_{f}$ for the period of $F-$ periodic chain is cyclic, i.e. $L_{f}\left(i_{1}, i_{2}\right)=i_{1}, L_{f}\left(i_{2}, i_{1}\right)=i_{2},\left(i_{1}, i_{2}\right) \in \mathbb{I}$. 
Corollary 6.3. Examples of cycles of admissible triplets defining $U$ and $D$ periodic tetrahedral chains are displayed in Table 4.

\begin{tabular}{|c|c|}
\hline$U$-chains & $D$-chains \\
\hline$(3,2,1) \rightarrow(2,1,4) \rightarrow(1,4,3) \rightarrow(4,3,2)$ & $(2,1,4) \rightarrow(1,4,3) \rightarrow(4,3,2) \rightarrow(3,2,1)$ \\
\hline$(4,3,1) \rightarrow(3,1,2) \rightarrow(1,2,4) \rightarrow(2,4,3)$ & $(3,1,2) \rightarrow(1,2,4) \rightarrow(2,4,3) \rightarrow(4,3,1)$ \\
\hline$(2,4,1) \rightarrow(4,1,3) \rightarrow(1,3,2) \rightarrow(3,2,4)$ & $(4,1,3) \rightarrow(1,3,2) \rightarrow(3,2,4) \rightarrow(2,4,1)$ \\
\hline$(3,4,2) \rightarrow(4,2,1) \rightarrow(2,1,3) \rightarrow(1,3,4)$ & $(4,2,1) \rightarrow(2,1,3) \rightarrow(1,3,4) \rightarrow(3,4,2)$ \\
\hline$(4,1,2) \rightarrow(1,2,3) \rightarrow(2,3,4) \rightarrow(3,4,1)$ & $(1,2,3) \rightarrow(2,3,4) \rightarrow(3,4,1) \rightarrow(4,1,2)$ \\
\hline$(4,2,3) \rightarrow(2,3,1) \rightarrow(3,1,4) \rightarrow(1,4,2)$ & $(2,3,1) \rightarrow(3,1,4) \rightarrow(1,4,2) \rightarrow(4,2,3)$ \\
\hline$(1,4,3) \rightarrow(4,3,2) \rightarrow(3,2,1) \rightarrow(2,1,4)$ & $(4,3,2) \rightarrow(3,2,1) \rightarrow(2,1,4) \rightarrow(1,4,3)$ \\
\hline$(1,2,4) \rightarrow(2,4,3) \rightarrow(4,3,1) \rightarrow(3,1,2)$ & $(2,4,3) \rightarrow(4,3,1) \rightarrow(3,1,2) \rightarrow(1,2,4)$ \\
\hline$(1,3,2) \rightarrow(3,2,4) \rightarrow(2,4,1) \rightarrow(4,1,3)$ & $(3,2,4) \rightarrow(2,4,1) \rightarrow(4,1,3) \rightarrow(1,3,2)$ \\
\hline$(2,1,3) \rightarrow(1,3,4) \rightarrow(3,4,2) \rightarrow(4,2,1)$ & $(1,3,4) \rightarrow(3,4,2) \rightarrow(4,2,1) \rightarrow(2,1,3)$ \\
\hline$(2,3,4) \rightarrow(3,4,1) \rightarrow(4,1,2) \rightarrow(1,2,3)$ & $(3,4,1) \rightarrow(4,1,2) \rightarrow(1,2,3) \rightarrow(2,3,4)$ \\
\hline$(3,1,4) \rightarrow(1,4,2) \rightarrow(4,2,3) \rightarrow(2,3,1)$ & $(1,4,2) \rightarrow(4,2,3) \rightarrow(2,3,1) \rightarrow(3,1,4)$ \\
\hline
\end{tabular}

Table 4: Cycles of admissible triplets defining $U$ and $D$ periodic tetrahedral chains.

Example 6.4. Using the cycles of Table 4 the numerical representation for $U$ - periodic tetrahedral chains with the initial $\left(i_{1}, i_{2}\right)=(3,2)$ is written in the form

$$
L_{u}(3,2)=1, L_{d}(2,1)=4, L_{u}(1,4)=3, L_{d}(4,3)=2
$$

And the corresponding composition defining the principal operator $M_{U}=$ $\bar{R}_{4} \bar{R}_{1} \bar{R}_{2} \bar{R}_{3}$ reconstructs this periodic chain geometrically.

For $L_{*}$-sequence of $U$ ( $D$ resp.) periodic tetrahedral chain we associate a corresponding operator $M_{U}=\bar{R}_{i_{4}} \cdots \bar{R}_{i_{1}}$ ( $M_{D}$ resp.) such that the $U$ periodic chain is a composition of $M_{U}$ ( $M_{D}$ resp.). Here $M_{F}=\bar{R}_{i_{2}} \bar{R}_{i_{1}}$.

Recall $\mathbb{I}=\left\{(\alpha, \beta) \in \Delta_{4} \times \Delta_{4}: \alpha \neq \beta\right\}, \quad \# \mathbb{I}=12$. Any two consecutive indexes $i_{k}, i_{k+1}$ determine uniquely the third one $i_{k+2}$ for an element of the chain of type $D, F, U$, and so on for subsequent pair $i_{k+1}, i_{k+2}$. Then we naturally define the corresponding maps

$$
\mathcal{L}_{u}, \mathcal{L}_{d}, \mathcal{L}_{f}: \mathbb{I} \rightarrow \mathbb{I}
$$

$\mathcal{L}_{*}\left(i_{1}, i_{2}\right)=\left(i_{2}, L_{*}\left(i_{1}, i_{2}\right)\right), * \in\{d, f, u\} . \# \mathbb{I}=12$ and on the basis of Table 1 $\mathcal{L}_{*}$ are bijections of $\mathbb{I}$. 
For any word $W_{m}$ of length $m$ we define the mapping $\mathcal{L}_{W_{m}}: \mathbb{I} \rightarrow \mathbb{I}$, $\mathcal{L}_{W_{m}}\left(i_{1}, i_{2}\right)=\left(i_{m+1}, i_{m+2}\right)$ which is a composition of bijections, $\mathcal{L}_{u}, \mathcal{L}_{d}, \mathcal{L}_{f}$ in the order of the adjoint word $\bar{W}_{m}$.

$$
\mathcal{L}_{*} \cdots \mathcal{L}_{*}\left(i_{1}, i_{2}\right)=\left(i_{m+1}, i_{m+2}\right) .
$$

This composition of bijections will be called the $\mathcal{L}$-sequence.

Example 6.5. Any word, say $W_{5}=D U U F D$, is represented by $\mathcal{L}$-sequence. Taking its adjoint word $I(D U U F D)=d d u f d$ we write

$$
\mathcal{L}_{W_{5}}=\mathcal{L}_{d} \mathcal{L}_{f} \mathcal{L}_{u} \mathcal{L}_{d} \mathcal{L}_{d}
$$

\begin{tabular}{|c||c|c|c|c|c|c|c|c|c|c|c|c|}
\hline$\left(i_{1}, i_{2}\right)$ & $(1,2)$ & $(1,3)$ & $(1,4)$ & $(2,1)$ & $(2,3)$ & $(2,4)$ & $(3,1)$ & $(3,2)$ & $(3,4)$ & $(4,1)$ & $(4,2)$ & $(4,3)$ \\
\hline \hline $\mathcal{L}_{W_{5}}\left(i_{1}, i_{2}\right)$ & $(1,3)$ & $(1,4)$ & $(1,2)$ & $(2,4)$ & $(2,1)$ & $(2,3)$ & $(3,2)$ & $(3,4)$ & $(3,1)$ & $(4,3)$ & $(4,1)$ & $(4,2)$ \\
\hline
\end{tabular}

Table 5: Table of the mapping $\mathcal{L}_{\text {duufd }}$.

There is a natural question, whether any periodic tetrahedral chain, with period $W_{m}$ is represented by a principal operator $M_{W_{m}}$, i.e. a finite composition of reflect-morphisms defined by a cycling $\mathcal{L}$-sequence $\mathcal{L}_{W_{m}}$, such that $\mathcal{L}_{W_{m}}\left(i_{1}, i_{2}\right)=\left(i_{1}, i_{2}\right)$. If we have the principal operator $M_{W_{m}}$ for a periodic chain, then any part of the chain of length $k n$ is represented by ordered composition of reflect-morphism in $M_{W_{m}}^{k}$.

Theorem 6.1. For any periodic tetrahedral chain with a period $W_{m}$ there exists a principal operator $M_{W_{m}}: \mathcal{T} \rightarrow \mathcal{T}$ consisting of a composition of reflect-morphisms $\bar{R}_{i}, i=1, \ldots, 4$, defined by a cycling composition of a numerical representation $\mathcal{L}_{W_{m}}$.

Proof. To each period $W_{m}$ we prescribe uniquely a bijection $\mathcal{L}_{W_{m}}$ of the twelve-element set $\mathbb{I}$. $\mathcal{L}_{W_{m}} \in S_{12}$, and as an element of the symmetric group has a finite order, say $k$ such that $\mathcal{L}_{W_{m}}^{k}=i d_{\mathbb{I}}$. Thus for any pair $\left(i_{1}, i_{2}\right) \in \mathbb{I}$ after $m k+2$ steps we get a cycle property.

A collection of $\mathcal{L}$-sequences is denoted by $\Gamma_{\mathcal{L}}$. To each element of $\Gamma_{\mathcal{T}}$ which can be treated also as an adjoint word, we associate uniquely a composition of reflect-morphisms $\bar{R}_{i_{n}} \cdots \bar{R}_{i_{1}}$ representing the corresponding configuration of $d, f, u$ elements in a chain. By construction of the sequence of 
numbers $i_{1}, \ldots, i_{n}$ in the reflect-morphism representation (see Section 2) the elements of $\Gamma_{\mathcal{T}}$ are indexed uniquely by the finite $\mathcal{L}$-sequences belonging to $\Gamma_{\mathcal{L}}$. These sequences are built of three bijections $\mathcal{L}_{u}, \mathcal{L}_{d}, \mathcal{L}_{f}$ which are elements of the permutation group $S_{12}$, i.e there is a well defined map $\Gamma_{\mathcal{T}} \rightarrow \Gamma_{\mathcal{L}}$ of finite words of $\Gamma_{\mathcal{T}}$ into the space of finite formal sequences of these three bijections. Taking the final composition of the corresponding $\mathcal{L}$ - sequence elements as elements of the permutation group $S_{12}$, we get the indexing map, $\Phi: \Gamma_{\mathcal{L}} \rightarrow S_{12}$. To each tetrahedral chain an indexing map $\Phi$ associates bijection $\mathbb{I} \rightarrow \mathbb{I}$. Image of an indexing map $\Phi$ is called the indexing space of $\Gamma_{\mathcal{T}}$.

Theorem 6.2. The indexing space of $\Gamma_{\mathcal{T}}$ is a tetrahedral group. $\Phi\left(\Gamma_{\mathcal{T}}\right)$ is a tetrahedral subgroup of $S_{12}$ generated by three elements,

$$
\mathcal{L}_{u}, \quad \mathcal{L}_{d}, \quad \mathcal{L}_{f}
$$

with the relations

$$
\mathcal{L}_{u}^{3}=i d, \quad \mathcal{L}_{d}^{3}=i d, \quad \mathcal{L}_{f}^{2}=i d, \quad\left(\mathcal{L}_{u} \mathcal{L}_{d}\right)^{2}=i d
$$

Elements $\mathcal{L}_{u}, \mathcal{L}_{d}$, with relations $\mathcal{L}_{u}^{3}=i d, \mathcal{L}_{d}^{3}=i d,\left(\mathcal{L}_{u} \mathcal{L}_{d}\right)^{2}=i d$ generate the tetrahedral group $T$ of 12 elements.

$$
T=\left\{i d, \mathcal{L}_{u}, \mathcal{L}_{d}, \mathcal{L}_{u}^{2}, \mathcal{L}_{d}^{2}, \mathcal{L}_{d} \mathcal{L}_{u}, \mathcal{L}_{u} \mathcal{L}_{d}, \mathcal{L}_{u}^{2} \mathcal{L}_{d}, \mathcal{L}_{u} \mathcal{L}_{d}^{2}, \mathcal{L}_{d}^{2} \mathcal{L}_{u}, \mathcal{L}_{d} \mathcal{L}_{u}^{2}, \mathcal{L}_{u} \mathcal{L}_{d}^{2} \mathcal{L}_{u}\right\}
$$

Example 6.6. To each tetrahedral chain, defined by a word $W_{m}$, the indexing map $\Phi$ associates a reduced word being an element of a tetrahedral group generated by $\mathcal{L}_{u}, \mathcal{L}_{d}, \mathcal{L}_{f}$ with the relations. For the word $W_{6}=$ $U F U F D D$ we have $I\left(W_{6}\right)=u f u f d u$ and $\Phi\left(I\left(W_{6}\right)\right)=\mathcal{L}_{u} \circ \mathcal{L}_{d} \circ \mathcal{L}_{f} \circ \mathcal{L}_{u} \circ$ $\mathcal{L}_{f} \circ \mathcal{L}_{u}=\mathcal{L}_{u}^{2}$. And finally an indexing word is equal to $U D$.

\section{Acknowledgements}

The authors wish to thank Stephen S.-T.Yau, Zbigniew Lonc, and Elizabeth Chen for helpful discussions and communications. 


\section{Appendix A. List of all 1-vertex clusters}

\begin{tabular}{|c|c|c|c|c|c|}
\hline \multicolumn{6}{|c|}{ Closed 1-vertex Clusters of Length 9} \\
\hline DUFDUF & UDFUDF & & & & \\
\hline \multicolumn{6}{|c|}{ Closed 1-vertex Clusters of Length 10} \\
\hline DUDFUDF & FUDUDFF & FDUDUFF & UDUFDUF & & \\
\hline \multicolumn{6}{|c|}{ Closed 1-vertex Clusters of Length 11} \\
\hline DUDUFDUF & DFUDUDFF & DFFUFDFF & FUDUFDUF & FUFDFFUD & FUFDFUDF \\
\hline FDUDFUDF & FDFUFDUF & FDFUFFDU & UDUDFUDF & UFFDFUFF & UFDUDUFF \\
\hline \multicolumn{6}{|c|}{ Closed 1-vertex Clusters of Length 12} \\
\hline DUDUDFUDF & DFUDUFDUF & DFUFDFUDF & DFUFDFFUD & FUDFUFDFF & FUFDUDUFF \\
\hline FUFFDFUFF & FFDUDFUDF & FFDFUFDUF & FFUDUFDUF & FFUFDFUDF & FDUFDFUFF \\
\hline FDFFUFDFF & FDFUDUDFF & UDUDUFDUF & UFDUDFUDF & UFDFUFFDU & UFDFUFDUF \\
\hline \multicolumn{6}{|c|}{ Closed 1-vertex Clusters of Length 13} \\
\hline DUDUDUFDUF & DUFDUDFUDF & DUFDUDFUFF & DUFDFUFFDU & DFUFFDFUFF & DFUFDUDUFF \\
\hline DFFUDUDUDF & DFFUDUFDUF & DFFUDUFDFF & DFFUFDUDFF & FUDUDUDFFU & FUDUDUFDUF \\
\hline FUDUFDFFUD & FUFDUDFUDF & FUFDFUFDUF & FUFDFUFFDU & FFDUDUDUFF & FFDFUDUDFF \\
\hline FFDFFUFDFF & FFUDUDUDFF & FFUFFDFUFF & FFUFDUDUFF & FDUDUDUFFD & FDUDUDFUDF \\
\hline FDUDFUFFDU & FDFUDUFDUF & FDFUFDFFUD & FDFUFDFUDF & UDUDUDFUDF & UDFUDUFDUF \\
\hline UDFUDUFDFF & UDFUFDFFUD & UFFDUDUDUF & UFFDUDFUDF & UFFDUDFUFF & UFFDFUDUFF \\
\hline UFDFUDUDFF & UFDFFUFDFF & & & & \\
\hline \multicolumn{6}{|c|}{ Closed 1-vertex Clusters of Length 14} \\
\hline DUDUDUDFUDF & DUDUDUFFDFF & DUDUFDFFUFF & DUDFUDUFDUD & DUDFUDUFDUF & DUDFUFDFFUD \\
\hline DUDFFUFFDFF & DUFDFFUFFDU & DUFDFFUFDFF & DUFDFUDUDFF & DUFDFUDUFFD & DFUDUDUFDUF \\
\hline DFUDUDUFDFF & DFUDUFDFFUD & DFUDFUFDUDF & DFUFDUDFUDF & DFUFDFUFFDU & DFUFDFUFDUF \\
\hline DFFUDUFDFUD & DFFUFDUDUFF & DFFUFDFUFDF & DFFUFFDFUFF & DFFUFFDFFUD & FUDUFDFUDFU \\
\hline FUDFUDUFDUF & FUDFUDUFDFF & FUDFUFDUDFF & FUFDUDFFUFD & FUFFDUDFUDF & FUFFDUDFUFF \\
\hline FUFFDFUDUFF & FUFFDFFUDUD & FFDUDUDFUDF & FFDUDFUFDUF & FFDFUDUFDUF & FFDFUFDFUDF \\
\hline FFDFUFDFUFF & FFDFUFDFFUD & FFUDUDUFDUF & FFUDUFDFUDF & FFUFDUDFUDF & FFUFDFUFFDU \\
\hline FFUFDFUFDUF & FFUFDFUFDFF & FDUDFUFDUFD & FDUFDUDFUDF & FDUFDUDFUFF & FDUFDFUDUFF \\
\hline FDFFUDUFDUF & FDFFUDUFDFF & FDFFUFFDUDU & FDFFUFDUDFF & FDFUDUFFDFU & UDUDUDUFDUF \\
\hline UDUDUDFFUFF & UDUDFUFFDFF & UDUFFDFFUFF & UDUFDUDFUDU & UDUFDUDFUDF & UDUFDFUFFDU \\
\hline UDFUFDUDUFF & UDFUFDUDFFU & UDFUFFDFUFF & UDFUFFDFFUD & UFFDUDFUFDU & UFFDFFUFFDU \\
\hline UFFDFFUFDFF & UFFDFUDUDFF & UFFDFUFDFUF & UFDUDUDFUDF & UFDUDUDFUFF & UFDUDFUFFDU \\
\hline UFDUFDFUDUF & UFDFUDUFDUF & UFDFUFDFUDF & UFDFUFDFFUD & & \\
\hline \multicolumn{6}{|c|}{ Closed 1-vertex Clusters of Length 15} \\
\hline DUDUDUDFFUFF & DUDUDFUFFDUD & DUDFUFFDFFUD & DUDFUFFDFUFF & DUDFUFDUDUFF & DUDFUFDUFDFF \\
\hline DUDFFUFDUDUD & DUFDUDUDFUDF & DUFDUDUDFUFF & DUFDFFUFDUFD & DUFDFUFDFFUD & DUFDFUFDFUDF \\
\hline DUFFDFUDUDFF & DUFFDFUFDFUF & DUFFDFFUFDUF & DUFFDFFUFDFF & DFUDUFDUDFFU & DFUFFDUDFUDF \\
\hline DFUFFDFFUDUD & DFUFDUDFFUFD & DFUFDUDFFUFF & DFUFDFUFFDFF & DFFUDUDUFDUF & DFFUFDFUFDUF \\
\hline DFFUFDFUFFDU & DFFUFFDFUDFU & FUDUDUDUFDUF & FUDUDUFDFFUD & FUDUDFUFFDFF & FUDUFDUDFUDF \\
\hline FUDUFDFUFFDU & FUDUFFDFFUFF & FUDFUFFDFFUD & FUDFUFFDFUFF & FUDFUFDUDUFF & FUFDUDUDFUDF \\
\hline FUFDUDFUFFDU & FUFDUFDFFUFF & FUFDFFUFFDFF & FUFDFUDUFDUF & FUFDFUFDFFUD & FUFFDFUDUDFF \\
\hline FUFFDFUDFUFD & FUFFDFFUFDFF & FUFFDFFUFDFU & FUFFDFFUFFDU & FFDFFUDUFDUF & FFDFFUDUFDFF \\
\hline FFDFFUFDUDFF & FFUFFDUDFUDF & FFUFFDUDFUFF & FFUFFDFUDUFF & FDUDUDUDFUDF & FDUDUDFUFFDU \\
\hline FDUDUFDFFUFF & FDUDFFUFFDFF & FDUDFUDUFDUF & FDUDFUFDFFUD & FDUFDFUDUDFF & FDUFDFFUFDFF \\
\hline FDUFDFFUFFDU & FDFFUFFDFFUD & FDFFUFFDFUFD & FDFFUFFDFUFF & FDFFUFDUDUFF & FDFFUFDUFDFU \\
\hline FDFUDUDUFDUF & FDFUDUFDFFUD & FDFUDFUFFDFF & FDFUFDUDFUDF & FDFUFDFUFFDU & FDFUFFDFFUFF \\
\hline UDUDUDUFFDFF & UDUDUFDFFUDU & UDUFFDFUDUDU & UDUFDFUDUDFF & UDUFDFUDFUFF & UDUFDFFUFDFF \\
\hline UDUFDFFUFFDU & UDFFUFFDFUDF & UDFFUFFDFUFF & UDFFUFDUDUFF & UDFFUFDFUFDF & UDFUDUDUFDUF \\
\hline UDFUDUDUFDFF & UDFUFDFUFDUF & UDFUFDFUFFDU & UDFUFFDFUDFU & UFFDUDUDFUDF & UFFDFFUFDUFD \\
\hline UFFDFUFDFFUD & UFFDFUFDFUDF & UFDUDFUDUFFD & UFDFUDUFFDFF & UFDFUDUFFDFU & UFDFUFDFFUFF \\
\hline UFDFFUDUFDUF & UFDFFUFFDUDU & & & & \\
\hline \multicolumn{6}{|c|}{ Closed 1-vertex Clusters of Length 16} \\
\hline DUDUFDFFUFDFF & DUDUFDFUDUDFF & DUDUFDFUDFUFF & DUDFUDUFFDFUD & DUDFFUFDUDUFF & DUDFFUFDUFDFF \\
\hline DUDFFUFFDFUFF & DUFDUDFFUFFDU & DUFDUDFUDUFFD & DUFDFFUDUFDUD & DUFDFFUDUFDUF & DUFDFUFDFFUFF \\
\hline DUFFDFUDUDUFF & DUFFDFUFDFUDF & DUFFDFUFDFFUD & DFUDUDUDUFDUF & DFUDUFFDFFUFF & DFUDUFFDFUDFU \\
\hline DFUDUFDFUFFDU & DFUDFUFDUDUFF & DFUDFUFFDFUDF & DFUDFUFFDFUFF & DFUFFDUDFUDUF & DFUFFDFFUFFDU \\
\hline DFUFFDFFUFDFU & DFUFFDFFUFDFF & DFUFFDFUDUDFF & DFUFFDFUDFUFF & DFUFFDFUDFUFD & DFFUDUDUFDFFU \\
\hline DFFUFDUDUDFFU & DFFUFDUFDFFUD & DFFUFFDUDFUDU & DFFUFFDUDFUDF & DFFUFFDUDFUFF & DFFUFFDFUDUFF \\
\hline FUDUDUDUFFDFF & FUDUFDUDFFUFF & $\begin{array}{l}\text { FUDUFDUDFFUFD } \\
\text { FUDU }\end{array}$ & FUDUFDFFUFFDU & FUDUFFDFFUDUD & FUDFUDUDUFDUF \\
\hline FUDFUDUDUFDFF & FUDFUFFDFUDFU & FUDFUFDFUFFDU & FUDFUFDFUFDUF & FUFDUDUDFFUFF & FUFDFFUFFDUDU \\
\hline FUFDFUDUFFDFU & FUFDFUDUFFDFF & FUFFDUDUDFUDF & FUFFDUDUDFUFF & FUFFDFUFDFUDF & FUFFDFUFDFFUD \\
\hline FUFFDFFUFDUFD & FFDUDUDUDFUDF & FFDUDUDFUFFDU & FFDUDUFDFFUFF & FFDUDFUDUFDUF & FFDUDFUFDFFUD \\
\hline FFDUDFFUFFDFF & FFDFUDUDUFDUF & FFDFUDUFDFFUD & FFDFUDFUFFDFF & FFDFUFFDFFUFF & FFDFUFDUDFUDF \\
\hline FFDFFUFDUDUFF & FFDFFUFFDFUFF & FFDFFUFFDFFUD & FFUDUDUDUFDUF & FFUDUDUFDFFUD & FFUDUDFUFFDFF \\
\hline FFUDUFFDFFUFF & FFUDUFDUDFUDF & FFUDUFDFUFFDU & FFUFFDFFUFFDU & FFUFFDFFUFDFF & FFUFFDFUDUDFF \\
\hline FFUFDUDUDFUDF & FFUFDUDFUFFDU & FFUFDUFDFFUFF & FFUFDFUDUFDUF & FFUFDFFUFFDFF & FDUDUDUDFFUFF \\
\hline FDUDFFUFFDUDU & FDUDFUDUFFDFU & FDUDFUDUFFDFF & FDUDFUFFDFFUD & FDUFDUDUDFUDF & FDUFDUDUDFUFF \\
\hline FDUFDFUFDFUDF & FDUFDFUFDFFUD & FDUFDFFUFDUFD & FDFFUDUDUFDUF & FDFFUDUDUFDFF & FDFFUFFDFUDFU \\
\hline FDFFUFDFUFFDU & FDFFUFDFUFDUF & FDFUDUDUFFDFF & FDFUFDUDFFUFF & FDFUFDUDFFUFD & FDFUFFDFFUDUD \\
\hline UDUDFUFDUDUFF & UDUDFUFDUFDFF & UDUDFUFFDFUFF & UDUFFDFFUFDFF & UDUFFDFUDUDFF & UDUFFDFUDFUFF \\
\hline UDUFDUDFFUFDU & UDFFUFDUDUDFF & UDFFUFDFUFFDU & UDFFUFDFUFDUF & UDFUDUFDUDFFU & UDFUDUFFDFFUD \\
\hline UDFUFDFUFFDFF & UDFUFFDUDFUDU & UDFUFFDUDFUDF & UFFDUDUDFUFFD & UFFDFFUDUFDUD & UFFDFFUDUFDUF \\
\hline UFFDFFUDUFDFF & UFFDFFUFDUDFF & UFFDFUDUDUFFD & UFFDFUDFUFFDU & UFDUDUDUDFUDF & UFDUDFUFDFFUD \\
\hline UFDUDFFUFDUFD & UFDUDFFUFFDFF & UFDUFDFFUFDUF & UFDUFDFFUFDFF & UFDUFDFUDUDFF & UFDFFUDUFDUDF \\
\hline UFDFFUFDUDUFF & UFDFFUFDUFDFU & UFDFFUFDUFDFF & UFDFFUFFDFUFF & UFDFFUFFDFUFD & UFDFFUFFDFFUD \\
\hline
\end{tabular}




\begin{tabular}{|c|c|c|c|c|c|}
\hline \multicolumn{6}{|c|}{ Closed 1-vertex Clusters of Length 17} \\
\hline DUDUFDFFUDUFFD & DUDUFFDFFUDUFF & DUDUFFDFFUFDFF & DUDFFUFDUFDFUD & DUDFFUFFDUDFFU & DUDFFUFFDFUFDF \\
\hline DUFDUDUDUDFUDF & DUFDUDFFUFFDFF & DUFDUDFFUFDUFD & DUFDUDFUFDFFUD & DUFDFFUDUFFDFF & DUFDFFUDUFFDFU \\
\hline DUFDFFUFFDFFUD & DUFDFUDFUFFDUD & DUFFDFUDFUFDUF & DUFFDFUDFUFFDU & DUFFDFFUDUFDUF & DUFFDFFUDUFDFF \\
\hline DUFFDFFUFDUDFF & DUFFDFFUFDFUFF & DFUDUDUDUFFDFF & DFUDUDUFFDFFUD & DFUDUFFDFFUDUD & DFUDFUFDUFDFFU \\
\hline DFUFFDFFUDUFFD & DFUFDFFUFFDUDU & DFFUDUDUDUFDUF & DFFUDUFDUDFUDF & DFFUDUFDFUFFDU & DFFUDUFFDFFUFF \\
\hline DFFUFDUDFUFFDU & DFFUFDUFDFUDFU & DFFUFDUFDFFUFF & DFFUFDFUDUFDUF & DFFUFDFUFFDFFU & DFFUFFDUDFFUFD \\
\hline DFFUFFDFFUFFDU & FUDUDFUFFDFUFF & FUDUDFUFDUDUFF & FUDUDFUFDUFDFF & FUDUFFDFUDUDFF & FUDUFFDFUDFUFD \\
\hline FUDUFFDFUDFUFF & FUDUFFDFFUFDFF & FUDUFFDFFUFDFU & FUDFUDUFFDFFUD & FUDFUDUFDUDFFU & FUDFUFFDUDFUDF \\
\hline FUDFUFDUFDFFUD & FUDFUFDFUFFDFF & FUFDUFDFUDUDFF & FUFDUFDFUDFUFF & FUFDUFDFFUFDFF & FUFDFFUFFDFUFD \\
\hline FUFDFFUFFDFUFF & FUFDFFUFDUDUFF & FUFDFFUFDUFDFF & FUFDFFUFDUFDFU & FUFFDUDFUDUFFD & FUFFDUDFFUFDUD \\
\hline FUFFDUDFFUFFDU & FUFFDFUDFUFFDU & FUFFDFUFDFFUFF & FUFFDFFUDUFDUD & FUFFDFFUDUFDUF & FFDUDUDUDFFUFF \\
\hline FFDUDFUDUFFDFF & FFDUDFUFFDFFUD & FFDUDFFUFFDUDU & FFDFUDUDUFFDFF & FFDFUFFDFFUDUD & FFDFUFDUDFFUFD \\
\hline FFDFUFDUDFFUFF & FFDFUFDFFUFFDU & FFDFFUDUDUFDUF & FFDFFUDUDUFDFF & FFDFFUFDFUFDUF & FFDFFUFDFUFFDU \\
\hline FFDFFUFFDFUDFU & FFUDUDUDUFFDFF & FFUDUFFDFFUDUD & FFUDUFDUDFFUFF & FFUDUFDFFUFFDU & FFUFFDUDUDFUDF \\
\hline FFUFFDUDUDFUFF & FFUFFDFFUFDUFD & FFUFFDFUFDFFUD & FFUFFDFUFDFUDF & FFUFDUDUDFFUFF & FFUFDFUDUFFDFF \\
\hline FFUFDFUDUFFDFU & FFUFDFUFFDFFUD & FFUFDFFUFFDUDU & FDUDUFDFUDUDFF & FDUDUFDFUDFUFF & FDUDUFDFFUFDFF \\
\hline FDUDFFUFFDFUFD & FDUDFFUFFDFUFF & FDUDFFUFDUDUFF & FDUDFFUFDUFDFF & FDUDFFUFDUFDFU & FDUFDUDFUDUFFD \\
\hline FDUFDUDFFUFFDU & FDUFDFUDFUFFDU & FDUFDFUFDFFUFF & FDUFDFFUDUFDUF & FDFFUDUFFDFFUD & FDFFUDUFFDFUDU \\
\hline FDFFUDUFDUDFFU & FDFFUFFDUDFUDU & FDFFUFFDUDFUDF & FDFFUFDUFDFFUD & FDFFUFDFUFFDFF & FDFUDFUFFDFUFF \\
\hline FDFUDFUFDUDUFF & FDFUDFUFDUFDFF & FDFUFFDFUDUDFF & FDFUFFDFUDFUFD & FDFUFFDFUDFUFF & FDFUFFDFFUFDFF \\
\hline FDFUFFDFFUFDFU & UDUDFFUFFDUDFF & UDUDFFUFFDFUFF & UDUDFUFFDUDFFU & UDUFFDFFUDUFFD & UDUFFDFFUFDFUF \\
\hline UDUFFDFUDFUFDU & UDFFUFFDUDFUDF & UDFFUFFDUDFUFF & UDFFUFFDFUDUFF & UDFFUFFDFUFDFF & UDFFUFDUFDFFUD \\
\hline UDFFUFDUFDFUDF & UDFUDUDUDUFDUF & UDFUDUFDFUFFDU & UDFUDUFFDFUDFU & UDFUDUFFDFFUFF & UDFUFDUFDFFUDU \\
\hline UDFUFFDUDFFUFD & UDFUFFDUDFFUFF & UDFUFFDFFUFFDU & UFFDUDUDUDFUDF & UFFDUDFFUFFDFF & UFFDUDFUDUFDUF \\
\hline UFFDUDFUFDFFUD & UFFDFFUDUFFDFU & UFFDFFUFFDFFUD & UFFDFUDUFDFFUD & UFFDFUDFUFFDFF & UFFDFUDFUFDUFD \\
\hline UFFDFUFDUDFUDF & UFFDFUFDFFUFFD & UFDUDUDUDFFUFF & UFDUDUDFFUFFDU & UFDUDFFUFFDUDU & UFDUFDFUDFUFFD \\
\hline UFDFUFFDFFUDUD & UFDFFUFFDUDFFU & & & & \\
\hline \multicolumn{6}{|c|}{ Closed 1-vertex Clusters of Length 18} \\
\hline DUDUDFFUFFDUDUD & DUDUFFDFFUFDFUF & DUDFUDUFFDFFUFF & DUDFUFFDUDFFUFD & DUDFFUFFDUDFUFF & DUFDUDUDUDFFUFF \\
\hline DUFDUDUDFFUFFDU & DUFFDFUDUFDFFUD & DUFFDFUDFUFFDFF & DUFFDFUFDUDFUDF & DUFFDFUFDFFUFFD & DUFFDFFUFFDFFUD \\
\hline DFUDFUFFDUDFUDF & DFUFFDUDFFUFDUD & DFUFFDUDFFUFDUF & DFUFDUFDFFUDUFF & DFUFDUFDFFUFDFF & DFUFDFFUFFDUDFF \\
\hline DFUFDFFUFFDFUFF & DFUFDFFUFFDFUFD & DFFUDUDUDUFFDFF & DFFUDUFDUDFFUFF & DFFUFDFUDUFFDFU & DFFUFDFUDUFFDFF \\
\hline DFFUFFDUDUDFUDF & DFFUFFDFUFDFFUD & DFFUFFDFFUFDUFD & DFFUFFDFFUFFDFF & FUDUDUFFDFFUDUD & FUDUDFUFFDUDFFU \\
\hline FUDUFFDFFUDUFFD & FUDFUDUDUDUFDUF & FUDFUDUFFDFFUFF & FUDFUDUFFDFUDFU & FUDFUDUFDFUFFDU & FUDFUFFDUDFFUFF \\
\hline FUDFUFFDUDFFUFD & FUDFUFFDFFUFFDU & FUFDUDFFUFFDUDU & FUFDUFDFFUDUFFD & FUFDFFUFFDUDFFU & FUFDFUFFDFFUDUD \\
\hline FUFFDUDUDUDFUDF & FUFFDUDFUFDFFUD & FUFFDUDFFUFDUFD & FUFFDUDFFUFFDFF & FUFFDFFUDUFFDFU & FUFFDFFUDUFFDFF \\
\hline FUFFDFFUFFDFFUD & FFDUDUFDFFUFDFF & FFDUDUFDFUDUDFF & FFDUDUFDFUDFUFF & FFDUDFFUFDUDUFF & FFDUDFFUFDUFDFU \\
\hline FFDUDFFUFDUFDFF & FFDUDFFUFFDFUFF & FFDUDFFUFFDFUFD & FFDFUDFUFDUDUFF & FFDFUDFUFDUFDFF & FFDFUDFUFFDFUFF \\
\hline FFDFUFFDFFUFDFU & FFDFUFFDFFUFDFF & FFDFUFFDFUDUDFF & FFDFUFFDFUDFUFF & FFDFUFFDFUDFUFD & FFDFFUDUFDUDFFU \\
\hline FFDFFUDUFFDFFUD & FFDFFUFDUFDFFUD & FFDFFUFDFUFFDFF & FFDFFUFFDUDFUDU & FFDFFUFFDUDFUDF & FFUDUDFUFDUDUFF \\
\hline FFUDUDFUFDUFDFF & FFUDUDFUFFDFUFF & FFUDUFFDFFUFDFU & FFUDUFFDFFUFDFF & FFUDUFFDFUDUDFF & FFUDUFFDFUDFUFF \\
\hline FFUDUFFDFUDFUFD & FFUFFDUDFFUFFDU & FFUFFDUDFUDUFFD & FFUFFDFFUDUFDUD & FFUFFDFFUDUFDUF & FFUFFDFUDFUFFDU \\
\hline FFUFFDFUFDFFUFF & FFUFDUFDFFUFDFF & FFUFDUFDFUDUDFF & FFUFDUFDFUDFUFF & FFUFDFFUFDUDUFF & FFUFDFFUFDUFDFU \\
\hline FFUFDFFUFDUFDFF & FFUFDFFUFFDFUFF & FFUFDFFUFFDFUFD & FDUDUDFFUFFDUDU & FDUDUFDFFUDUFFD & FDUDFFUFFDUDFFU \\
\hline FDUFDUDUDUDFUDF & FDUFDUDFUFDFFUD & FDUFDUDFFUFDUFD & FDUFDUDFFUFFDFF & FDUFDFFUDUFFDFU & FDUFDFFUDUFFDFF \\
\hline FDUFDFFUFFDFFUD & FDFFUDUDUDUFDUF & FDFFUDUFFDFFUFF & FDFFUDUFFDFUDFU & FDFFUDUFDFUFFDU & FDFFUFFDUDFFUFF \\
\hline FDFFUFFDUDFFUFD & FDFFUFFDFFUFFDU & FDFUDUFFDFFUDUD & FDFUDFUFFDUDFFU & FDFUFDFFUFFDUDU & FDFUFFDFFUDUFFD \\
\hline UDUDUFFDFFUDUDU & UDUDFFUFFDFUFDF & UDUFFDFFUDUFDFF & UDUFDUDFFUFFDFF & UDUFDFFUDUFFDFU & UDFFUFFDFFUFFDU \\
\hline UDFFUFDUDFUFFDU & UDFFUFDUFDFFUFF & UDFFUFDFUDUFDUF & UDFFUFDFUFFDFFU & UDFUDUDUDUFFDFF & UDFUDUDUFFDFFUD \\
\hline UFFDUDUDUDFFUFF & UFFDUDFUDUFFDFF & UFFDFFUDUDUFDUF & UFFDFFUFFDFFUFF & UFFDFFUFFDFUDFU & UFFDFFUFDFUFFDU \\
\hline UFFDFUFDUDFFUFF & UFFDFUFDUDFFUFD & UFDUFDFFUDUFDUF & UFDFUDFUFFDUDFF & UFDFUDFUFFDFUFF & UFDFUFFDFFUDUFF \\
\hline UFDFUFFDFFUFDFU & UFDFUFFDFFUFDFF & UFDFFUDUFFDFUDU & UFDFFUDUFFDFUDF & & \\
\hline \multicolumn{6}{|c|}{ Closed 1-vertex Clusters of Length 19} \\
\hline DUDUFFDFFUDUDUFF & DUDUFFDFFUDUFDFF & DUDFFUFFDUDUDFFU & DUFFDFUFDUDFFUFD & DUFFDFUFDUDFFUFF & DUFFDFFUDUDUFDUF \\
\hline DUFFDFFUFFDFUDFU & DUFFDFFUFFDFFUFF & DFUDFUFFDUDFFUFF & DFUDFUFFDFFUFFDU & DFUFFDUDFFUFFDFF & DFUFFDUDFUFDFFUD \\
\hline DFFUDUDUFFDFFUDU & DFFUDUFFDFUDUDFF & DFFUDUFFDFUDFUFF & DFFUDUFFDFFUFDFF & DFFUFFDUDFFUFFDU & FUDFUDUDUDUFFDFF \\
\hline FUDFUDUDUFFDFFUD & FUFFDUDUDUDFFUFF & FUFFDUDUDFFUFFDU & FFDUDUDFFUFFDUDU & FFDUDUFDFFUDUFFD & FFDUDFFUFFDUDFFU \\
\hline FFDFUDUFFDFFUDUD & FFDFUDFUFFDUDFFU & FFDFUFFDFFUDUFFD & FFDFFUDUDUDUFDUF & FFDFFUDUFDFUFFDU & FFDFFUDUFFDFUDFU \\
\hline FFDFFUDUFFDFFUFF & FFDFFUFFDUDFFUFD & FFDFFUFFDUDFFUFF & FFDFFUFFDFFUFFDU & FFUDUDUFFDFFUDUD & FFUDUDFUFFDUDFFU \\
\hline FFUDUFFDFFUDUFFD & FFUFFDUDUDUDFUDF & FFUFFDUDFFUFFDFF & FFUFFDUDFFUFDUFD & FFUFFDUDFUFDFFUD & FFUFFDFFUDUFFDFF \\
\hline FFUFFDFFUDUFFDFU & FFUFFDFFUFFDFFUD & FFUFDUDFFUFFDUDU & FFUFDUFDFFUDUFFD & FFUFDFFUFFDUDFFU & FDUFDUDUDUDFFUFF \\
\hline FDUFDUDUDFFUFFDU & FDFFUDUDUDUFFDFF & FDFFUDUDUFFDFFUD & UDUDFFUFFDUDUDFF & UDUDFFUFFDUDFUFF & UDUFFDFFUDUDUFFD \\
\hline UDFFUFFDUDUDFUDF & UDFFUFFDFFUFFDFF & UDFFUFFDFFUFDUFD & UDFFUFDFUDUFFDFF & UDFFUFDFUDUFFDFU & UFFDUDUDFFUFFDUD \\
\hline UFFDUDFFUFFDFUFF & UFFDUDFFUFDUDUFF & UFFDUDFFUFDUFDFF & UFFDFFUDUFFDFFUD & UFDUFDFFUDUFFDFF & UFDUFDFFUFFDFFUD \\
\hline UFDFFUDUFDFUFFDU & UFDFFUDUFFDFFUFF & & & & \\
\hline
\end{tabular}

DUFFDFFUDUDUFFDFF DUFFDFFUDUFFDFFUD DFFUDUFFDFFUDUFFD DFFUFFDUDUDFFUFFD FFDFFUDUDUDUFFDFF FFDFFUDUDUFFDFFUD FFUFFDUDUDUDFFUFF FFUFFDUDUDFFUFFDU UDFFUFFDUDUDFFUFF UDFFUFFDUDFFUFFDU UFFDUDFFUFFDUDFFU UFFDFFUDUDUFFDFFU

\section{References}

[1] H. S. M. Coxeter, Regular Polytopes. Dover, New York 1973.

[2] H. S. M. Coxeter, Close-packing and froth. Illinois J. Math., Vol. 2, Issue 4B (1958), 746-758.

[3] H. R. Farran, S. A. Robertson, Regular convex bodies. J. London Math. Soc. (2), 49 (1994), 371-384. 
[4] Hans E. Debrunner, Tiling Euclidean d-space with congruent symplexes. Annals of the New Yourk Academy of Sci., Vol. 440, (1985), 230-261.

[5] Xianfeng David Gu and Shing-Tung Yau, Computational Conformal Geometry. Advanced Lectures in Mathematics, Higher Edu. Press/Intl. Press of Boston, 2008.

[6] D. J. Hoylman, The densest lattice packing of tetrahedra. Bull. of the AMS, Vol. 76, (1970), 135-137.

[7] A. C. Hurley, Some Helical Structures Generated by Reflections. Aust. J. Phys., Vol. 38, (1985), 299-310.

[8] J. C. Lagarias and Ch. Zong, Mysteries in Packing Regular Tetrahedra. Notices of AMS, Vol. 59, No. 11, (2012), 1540-1549.

[9] J. H. Mason, Can regular tetrahedra be glued together face to face to form a ring? Math. Gaz., 56 (1972), 194-197.

[10] S. K. Stein, The Planes Obtainable by Gluing Regular Tetrahedra. The American Mathematical Monthly, Vol. 85, No. 6 (1978), 477-479.

[11] A. F. Wells, Survey of Tetrahedral Structures. Philosophical Transactions of the Royal Society of London. Series A, Mathematical and Physical Sciences, Vol. 319, No. 1548 (Aug. 28, 1986), pp. 291-335.

[12] Ch. Zheng, R. Hoffmann and D. R. Nelson, A Helical Face-Sharing Tetrahedron Chain with Irrational Twist, Stella Quadrangula, and Related Matters J. Am. Chem. Soc., Vol. 112, (1990), 3784-3791.

[13] Shing-Tung Yau and Steve Nadis, The Shape of Inner Space. Basic Books, New York, 2010. 
Wydzią MATEMATYKi I NAUK INFORMACYJNYCH PoliteChNIKA WARSZAWSKA

Pl. Politechniki 1, 00-661 Warszawa, Poland

E-mail address: babikerhb@gmail.com

Instytut MATEMATYCZNy PAN, UL. ŚNIADECKICH 8, 00-950 WARSZAWA AND Wydzią Matematyki I NAUK INFORMACYJNYCH PoliteChNika WARSZAWSKA

Pl. Politechniki 1, 00-661 Warszawa, Poland

E-mail address: janeczko@mini.pw.edu.pl

ReCEIVED July 20, 2015

AcCepted July 30, 2015 
